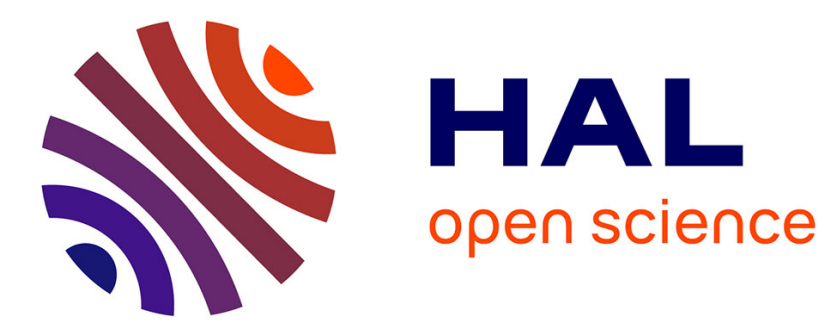

\title{
Biomolecular Recognition: The Current Challenge
}

Pierre Bongrand

\section{To cite this version:}

Pierre Bongrand. Biomolecular Recognition: The Current Challenge. A.R. Bizzari et R Cannistraro. Dynamic force spectroscopy and biomolecular recognition, CRC press - boca Raton, pp.1-50, 2012. inserm-01070064

\section{HAL Id: inserm-01070064 https://www.hal.inserm.fr/inserm-01070064}

Submitted on 30 Sep 2014

HAL is a multi-disciplinary open access archive for the deposit and dissemination of scientific research documents, whether they are published or not. The documents may come from teaching and research institutions in France or abroad, or from public or private research centers.
L'archive ouverte pluridisciplinaire HAL, est destinée au dépôt et à la diffusion de documents scientifiques de niveau recherche, publiés ou non, émanant des établissements d'enseignement et de recherche français ou étrangers, des laboratoires publics ou privés. 


\title{
Biomolecular Recognition: The Current Challenge
}

\author{
Pierre Bongrand \\ Lab. Adhesion and Inflammation, INSERM U600, CNRS U6212 \\ Parc Scientifique de Luminy \\ Case 937, 13288 Marseille Cedex 09, FRANCE \\ pierre.bongrand@inserm.fr, http://lai.sciences.univmed.fr
}

May 29, 2014

This author-edited text appeared in the book entitled: Dynamic Force Spectroscopy and biomolecular recognition, edited by A.R. Bizzari and $R$ Cannistraro, CRC Press, Boca-Raton, 2012, pp 1-50.

\section{Contents}

1 Introduction 2

2 What is the use of biomolecular interactions 4

2.1 Cell structure: statics and dynamics. . . . . . . . . . . . . . . 4

2.2 Cell differentiation . . . . . . . . . . . . . . . . . . 4

2.3 Cell adhesion . . . . . . . . . . . . . . . . . . . 5

2.4 Immune recognition . . . . . . . . . . . . . . . . . . . . . 7

2.5 Signal generation . . . . . . . . . . . . . . . . . . 9

3 Brief historical outline of recent investigations made on biomolecule recognition at the single bond level $\quad \mathbf{1 0}$

3.1 Studying bond rupture at the single molecule level . . . . . . . . . . . . 10

3.2 Measuring bond formation at the single molecule level . . . . . . . . . . 14

4 Which parameters do we need to account for biomolecule recognition?

4.1 The affinity constant . . . . . . . . . . . . . . . 16

4.2 Kinetic constants : the on-rate and the off-rate . . . . . . . . . . . 17

4.2.1 The force-dependent dissociation rates . . . . . . . . . . . 17

4.2.2 Distance-dependent association rates . . . . . . . . . . . . 19

4.3 Avidity of biomolecule interactions: an incompletely defined parameter 21

4.4 Specificity of biomolecule interactions: an essential property that is difficult to define accurately . . . . . . . . . . . . . . . . . . 22 
4.5 Ligand-receptor interactions are influenced by parameters that are extrinsic to both ligand and receptor molecules . . . . . . . . . . .

5 Relationship between biomolecule structure and recognition events 24

5.1 Intermolecular forces in the biological milieu . . . . . . . . . . 25

5.1 .1 Electrostatic forces . . . . . . . . . . . . . . . 25

5.1 .2 The hydrogen bond . . . . . . . . . . . . . . 26

5.1.3 Different timescale: electrodynamic interactions . . . . . . . . 27

5.1.4 Using the formalism of surface physical-chemistry. Hydrophobic bonds. . . . . . . . . . . . . . . . . . . . 28

5.2 General properties of ligand-receptor association . . . . . . . . . . 30

5.2.1 A static view of ligand-receptor complexes . . . . . . . . . . . . 30

5.2.2 Dynamics of ligand-receptor interaction . . . . . . . . . . . . 31

5.2.3 Mechanisms influencing the specificity of biomolecule interaction 33

5.3 Information yielded by computer simulation . . . . . . . . . . . 35

5.3.1 Limitations and technical advances. . . . . . . . . . . . . 37

5.3.2 New information on molecular association may be provided by computer simulations . . . . . . . . . . . . 38

6 Conclusion $\quad 39$

7 References $\quad 39$

\begin{abstract}
Biomolecules essentially fulfill their function through continual recognition of and binding to other molecules. Biomolecular recognition is therefore a phenomenon of prominent importance. When the progress of monoclonal antibody technology and genetic engineering allowed biologists to characterize and isolate an impressive variety of receptor molecules, it was first felt that affinity constants and kinetic rates provided a satisfactory account of receptor-ligand interactions. However, a number of advances that occurred during the last two decades showed that i) the conventional framework was not sufficient to predict the behaviour of biomolecules in many physiologically relevant situations, ii) a number of techniques allowed investigators to dissect biomolecule interactions at the single bond level and obtain new information on the kinetic and mechanical properties of these interactions, iii) new theoretical techniques and the development of computer simulation as well as the enormous increase of available structural data provided new avenues to relate structural and functional properties. The aim of this introductory chapter is to present a brief outline of these advances and pending issues.
\end{abstract}

\title{
1 Introduction
}

Life relies on myriads of interactions between the molecular components of living systems. Proteins are a remarkable example in view of their diversity (the very name of proteins stems from Proteus, a greek god known for his capacity to change shape). 
Several decades ago, the author of a well-known treatise on proteins (Creighton 1983) wrote that ... the biological function of proteins almost invariably depends on their direct physical interaction with other molecules. More recently, systematic use of powerful techniques such as yeast double hybrid or mass spectrometry was a basis for a large scale attempt to build exhaustive databases of protein interactions, the so-called interactome (Blow 2009). Over 250,000 interactions between about 22,000 proteins were recorded in the Unified Interactome Database on year 2008 (Chaurasia 2008).

Until recently, it seemed that the conventional concepts and methods used to study chemical equilibria provided a suitable framework to deal with biomolecular recognition. As reckoned two decades ago (Williams 1991), the concepts of specificity and affinity had seemed sufficient to deal with biological phenomena for many years, and only conventional kinetic constants had to be added to explain some recent findings. However, a number of reports supported the importance of forces in biological interactions (Capo 1978, Jaalouk 2009) and theoretical models of cell functions such as adhesion have included mechanical parameters (Bell 1984, Mege 1987). This was an incentive to devise experimental methods allowing us to study the response of biomolecules to forces with high temporal and spatial resolution, up to the single molecule level. Simultaneously, continuous progress in molecular dynamics allowed computer scientists to report on simulations of the response of biomolecules to external forces (Grubmuller 1996, Izrailev 1997, PuklinFaucher 2006), thus allowing deeper interpretation of experimental results (Florin 1994, Rief 1997). These advances were also facilitated by the tremendous increase of structural data on biomolecules, based on X ray cristallography and nuclear magnetic resonance, and the use of genetic engineering techniques to relate structural and functional data, as exemplified by alanine-scanning that consists of systematically replacing aminoacids with alanin in protein-protein interaction areas to obtain a direct estimate of their contribution to binding energy (Cunningham 1989). The development of dynamic force spectroscopy is a remarkable example of an innovative approach stemming for a number of different advances and yielding a new kind of information that might shed a new light on important and unresolved issues.

The goal of this chapter is to present as palatably as possible a number of biological processes and recent methodological advances that played an important role in the development of dynamic force spectroscopy and may benefit from this growing domain. The first section includes selected examples of biological situations that are heavily dependent on biomolecular recognition. This will be the basis for defining the questions we need to ask. The next section is a brief outline of recent progress done in the study of molecular interactions, particularly at the single bond level, which shaped the present state-of-the art. The next section is intended to define and analyze the parameters required to provide an adequate account of biomolecule interactions, i.e. to include the pieces of information that are needed to predict the behaviour of a given ligand-receptor couple under physiological conditions. The last section gives a brief description of the application of conventional physical-chemical knowledge and newer computer simulation methods to the study of links between biomolecule structure and association properties. Admittedly, the field of biomolecule interactions is too vast to be exhaustively discussed in the limited space available. Also, it is unavoidable that the topics selected in this chapter should reflect the limitations of the author's fields of 
competence and interest. Therefore, I apologize for the omission of many key references that would certainly have enriched this presentation.

\section{What is the use of biomolecular interactions}

The goal of this section is to describe several important biological processes in order to illustrate the role of biomolecule interactions and the constraints that must be met.

\subsection{Cell structure: statics and dynamics.}

Clearly, any living cell or organism would fall into pieces in absence of the molecular interactions linking their components. It is important to emphasize that both qualitative and quantitative properties of these interactions are essential. Thus, it is well recognized that cell formation requires an autoorganization capacity of biomolecules that must be able to bind to each other with sufficient specificity to avoid durable presence of potentially harmful molecular interactions (Vavouri 2009). In addition, the rheological properties of cells are considered to be driven by the properties of underlying cytokeletal elements, which are themselves dependent on the kinetic and mechanical properties of intermolecular associations (Wachsstock 1994). These points are important in view of the recently recognized importance of cell mechanics in situations of medical interest such as cancer cell metastasis (Glinsky 2003, Remmerbach 2009) or letal inflammatory processes such as the acute respiratory disease syndrome (Nishino 2005). Cell shape is considered to be highly dependent on the dynamic organization of a network of rodlike structures including actin microfilaments, tubulin microtubules and intermediate filaments. These are highly plastic structures whose growth or retraction is determined by a variety of interaction events, and particularly polymerization/depolymerization as a consequence of tunable kinetics of monomer association or dissociation. Other important events are movements driven by so-called motor molecules such as myosin or kinesin that are able to generate force-dependent displacements. Much effort was recently done to investigate the mechanisms of association/ dissociation and force generation by these molecules.

\subsection{Cell differentiation}

A remarkable feature of living cells composing complex organisms is their capacity to acquire different structural and functional capacity whereas they share a common set of genes. While the mechanisms of differentiation are not yet fully understood, a primary process is the selective synthesis of particular proteins as a consequence of gene activation by a combination of over 100 DNA binding proteins with a specificity for a number of regulatory sites on the DNA. Such a complex set of interactions remains incompletely known, but an extensive network of DNA/protein interactions clearly plays an important role in differentiation (Badis 2009). 


\subsection{Cell adhesion}

As previously reviewed (Pierres 2000), cell adhesion is a fundamental process that influences nearly all steps of cell function. Thus, cell survival and proliferation are often dependent on a strong attachment to solid surfaces, a phenomenon known as anchorage dependence (Folkman 1978, Chen 1997). An attractive interpretation of experimental findings was that cell adhesion might be required to induce marked cell flattening and spreading on the surface, and that cell behaviour might be shapesensitive (Pierres 2002, Neves 2008).

Cell migration on a surface is also highly dependent on the qualitative and quantitative properties of cell-surface interactions. It has long been shown that efficient cell migration required that binding strength, i.e. the mechanical force required to detach adherent cells, fell within a particular range (Palecek 1997). Too strongly adherent cells are expected to remain stuck on a fixed place (Jay 1995). In contrast, a minimal adhesion efficiency is probably required in order that a lamellipodium sent forward by a motile cell be able to remain stuck on the surface and drag forward the cell body with concomitant detachment of the rear part of the cell (Palecek 1998). More recently, it was reported that moving cells were able to probe the rigidity of underlying surfaces and move towards more rigid regions, a phenomenon called durotaxis (Lo 2000).

Cell differentiation is also strongly influenced by the properties of underlying surfaces. While this well-known phenomenon has long been interpreted by hypothesizing that cells were essentially sensitive to the biochemical structures of ligands exposed by surrounding surfaces and recognized by their receptors (Kaplan 1982), more recent experiments showed that cell responses were also dependent on the stiffness of these surfaces (Engler 2006). The mechanisms allowing cells to measure surface stiffness remain poorly understood, but it is likely that this involves the response to forces of surface biomolecules adhering to nearby ligands.

Indeed, cells continually probe their environment to adapt their shape, motion, and other functions such as proliferation or mediator release. Environment sensing may result from the uptake of soluble ligands by membranes. However, a more accurate and less noise-sensitive way of probing cellular environment may result from mechanical exploration through continual formation and retraction of protrusions such as lamellipodia (Dobereiner 2006) and finger-like filopodia (Faix 2006) or through transverse membrane undulations (Zidovska 2006, Pierres 2008, thus inducing transient contacts between membrane receptors and fixed ligands, which may provide a powerful way of rapidly gathering information (Pierres 2009). The outcome of interactions is heavily dependent on the kinetics of bond formation between surface-attached ligands and receptors, as well as the strengh of attachements. These phenomena are highly dependent on the kinetics and mechanics of receptor-ligand interactions. Arguably, cells use dynamic force spectroscopy to probe their environment (see Figure 1).

Inflammation is an ubiquitous process used by multicellular organisms to cope with various forms of aggression, and particularly infection. A key step is the adhesion of flowing blood leukocytes to the vessel walls, with subsequent transmigration through these walls and entry into tissues containing infectious agents or damaged cells. Unraveling the mechanisms of leukocyte interaction with endothelial cells coating the vessel 


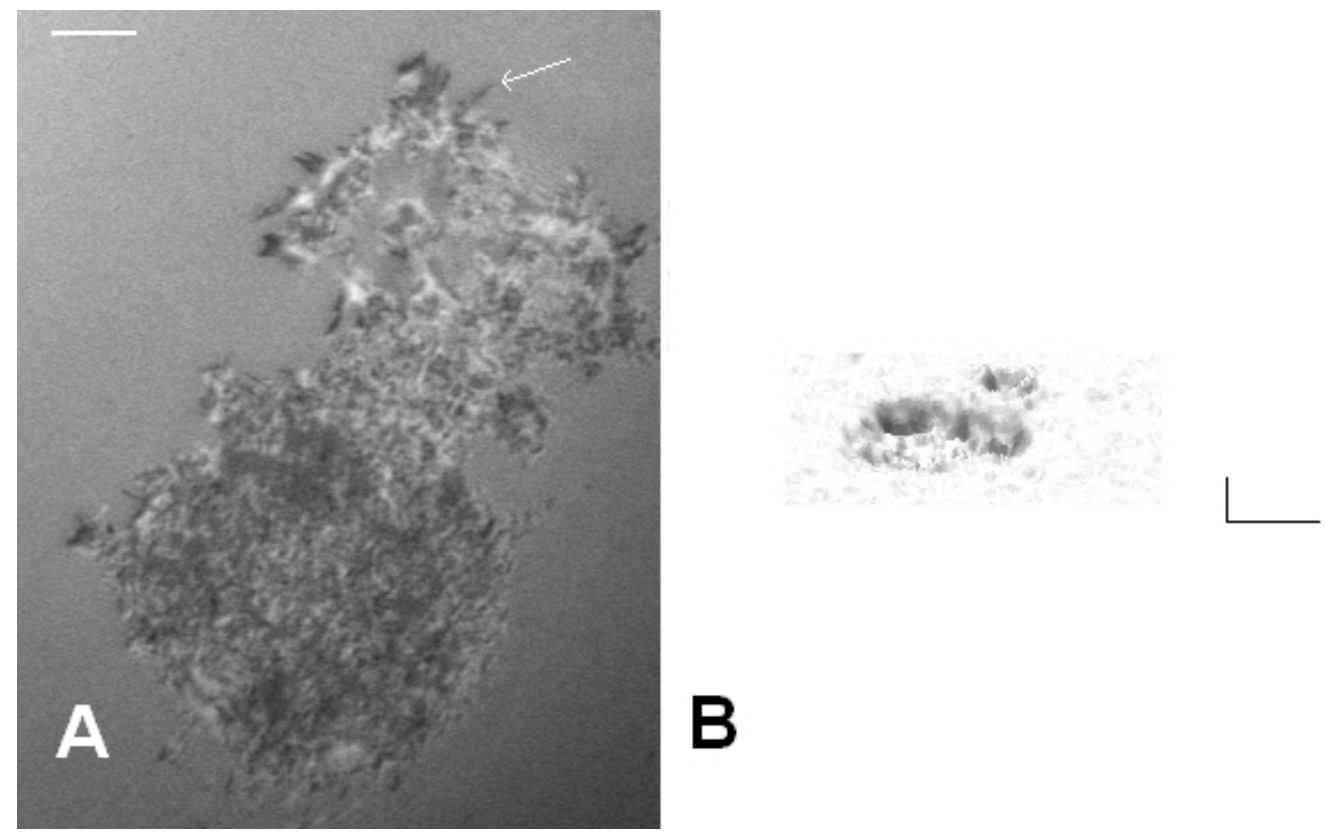

Figure 1: Cells probe their environment. A. A monocytic THP-1 cell was deposited on a surface coated with fibronectin and examined with interference-reflection contrast microscopy (IRM). Short filopodia (white arrow) appear as black lines. B. The image shows the underside of a lymphocyte falling on an activating surface, and tridimensional shape was derived from IRM images. A dynamic study revealed undulations of a few $\mathrm{nm}$ amplitude and $\mathrm{Hz}$ frequency. Horizontal bar length is $5 \mu \mathrm{m}$ and vertical bar length is $100 \mathrm{~nm}$. see (Cretel 2010) for details.

walls was a major task during the last two decades, and this provided a model of prominent biophysical interest (Fig. 2).

It has been known for more than a century that locally activated endothelial cells are able to bind to flowing leukocytes which undergo a nearly hundredfold velocity decrease (typically from $1 \mathrm{~mm} / \mathrm{s}$ to $10 \mu \mathrm{m} / \mathrm{s}$ ). Leukocytes then display a characteristically jerky motion called rolling. During the rolling phase, leukocytes remain sufficiently close to the wall to detect specific molecules with a capacity to activate strong leukocyte attachment and arrest. Displacement towards interendothelial junctions and migration to the peripheral tissues then follow. The progress of molecular biology and monoclonal antibody technology allowed identification of the adhesion molecules involved in leukocyte/endothelial interaction during the eighties, and the overall mechanisms of rolling and firm adhesion were disclosed in 1991 (Lawrence 1991, vonAndrian 1991). Briefly, proper stimulation of endothelial cells was shown to generate rapid expression of so-called selectin molecules on the membranes of endothelial cells. Thus, P-selectin that is stored in specialized granules may be externalized within minutes. P-selectin is a long (about $40 \mathrm{~nm}$ ) molecule the distal extremity of which bears a binding sites specific for characteristic structures bearing the sialyl - Lewis ${ }^{x}$ tetrasaccharide. This ligand is exposed on molecules borne by leukocyte membranes such as PSGL-1 (a 40 nm carbohydrate-rich molecule). The jerky rolling motion may be accounted for by a rapid formation and dissociation of P-selectin/PSGL-1 bonds as shown with model 


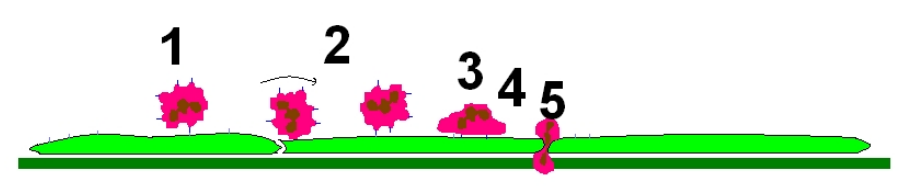

Figure 2: Leukocyte arrest on activated endothelium. Rapidly flowing leukocytes are first tethered by receptors such as P-selectin that appear on the membrane of activated endothelial cells (1). Then they begin rolling with a nearly hundredfold velocity decrease (2), which allows them to detect activating mediators such as chemokines on endothelial surfaces. These molecules activate leukocyte integrins, which results in firm cell adhesion (3). Then cells migrate towards endothelial cell junctions (4), and undergo impressive deformation that allows them to traverse the endothelial junctions. Finally, after crossing the basal membrane, they accede to inflamed tissues (5)

systems (Brunk 1996). During the rolling phase, leukocyte adhesion receptors belonging to the integrin family get activated by molecules linked to the endothelial cell pericellular matrix. These integrins then strongly bind to their ligand and induce a durable arrest. As an example, leukocyte integrin LFA-1 (which means lymphocyte function associated-1, also called CD11aCD18) will bind to ICAM-1 (intercellular cell adhesion molecule I, CD54) on endothelial cell surfaces. Flow chambers (see below) were used to study leukocyte arrest on planar surfaces coated with endothelial cell monolayers or molecules. A question that rapidly emerged consisted of understanding why P-selectin/PSGL-1 interaction resulted in rolling, whereas integrin/ligand association could not occur in absence of rolling, even if leukocyte integrins were activated before the experiment. Since the affinity of P-selectin/PSGL-1 and integrin/ligand interaction fell into the same range, it was soon suggested that P-selectin/PSGL-1 interaction might display peculiar physical properties, with high association and dissociation rates (allowing rapid cell attachment and detachment) and high mechanical strength to resist hydrodynamic forces (otherwise, these interactions would not generate any detectable cell arrest).

A general conclusion of these studies is that cell function is dependent on precise kinetic and mechanical properties of their adhesion receptors as well as tight regulation of these parameters.

\subsection{Immune recognition}

The immune system provides particularly important models of biological recognition. The task of immune cells consists of detecting foreign and potentially harmful particles or molecules in order to destroy them. Foreign particles may be pathogens, cancer 
cells or damaged cells that may release harmful metabolites. Immune recognition is of utmost importance and failure may entail devastating consequences. Indeed, a marked immune deficiency is known to result in letal infection within a few days or even hours after birth. Conversely, excessive immune activation may result in death as may be observed in allergic conditions or autoimmune diseases. It is probably because of this utmost importance that three complementary recognition mechanisms evolved and remained active in higher vertebrates.

Antibodies are protein molecules that may be generated by injecting animals with foreign substances that are consequently called antigens (which means: antibody generators). Antibodies share remarkable structural properties shared by the plasma proteins called immunoglobulins. Each antibody molecules possesses between 2 and 10 identical antigen binding sites called paratopes. There seems to be no limit to the recognition capacity of antibodies: they can specifically bind to proteins, carbohydrates, lipids, nucleic acids and even totally artificial structures such as dinitrophenol. Further, antibody efficiency is dependent on quantitative properties of binding sites such as affinity constant or association kinetics (Foote 1991, Diz 2008), as explained below. Antibodies bind antigens with an affinity constant that may be as high as $10^{10}-10^{12} M^{-1}$ and their specificity is illustrated by their capacity to discriminate between antignenic sites (called epitopes) differing by a single aminoacid. The study of antibodies was long made difficult by the high heterogeneity of antibodies raised after injecting animals with a given antigen. However, monoclonal antibodies provide a highly efficient basis for studying molecular recognition (Murphy 2008).

The specific antigen receptors born by $\mathrm{T}$ lymphocytes ( $\mathbf{T}$ cell receptors, or TCRs) represent a different recognition system. A major task of $\mathrm{T}$ lymphocytes consists of detecting cells containing foreign material such as viral proteins. The recognition principle is remarkable: most cells express on their surface on the order of 10,000 oligopeptides of 10-15 aminoacids nearly randomly sampled from the proteins they synthesize. Each oligopeptide appears as a few units bound to specialized membrane molecules encoded by genes belonging to the major histocompatibility complex (MHC) (Murphy 2008) It is remarkable that a T lymphocyte can detect a few or even a single foreign oligopeptide on a cell after scanning its membrane for 5-10 minutes (Bongrand 1998). Another remarkable point is that a number of studies strongly supported the hypothesis that the outcome of the recognition of a foreign oligopeptide by a T lymphocytes is dependent on the physical properties of TCR/ligand interaction. Indeed, the lifetime of individual TCR/ligand bonds might be a key determinant of T lymphocyte activation, since a too short interaction might result in cell paralysis rather than activation of effector functions (McKeithan 1995). Thus, quantifying these interactions between membrane-bound receptors and ligands is a current challenge of prominent importance (Huppa 2010, Huang 2010).

While the aforementioned two recognition mechanisms have been a focus of intense investigation during the last three or for decades, it is well recognized that the immune function also requires a set of so-called innate recognition mechanisms that are able to detect foreign microorganisms or damaged cells. Thus, a variety of receptors such as scavenger receptors (Greaves 2009) or toll-like receptors (Imler 2001) can detect remarkable structures such as double stranded RNAs that are not expressed by 
eukaryotic cells or denatured proteins and altered lipids that appear in damaged cells. The exquisite specificity of antibodies and TCRs may be responsible for the necessity of an additional recognition mechanism: since a given lymphocyte bears receptors of a single specificity (this is a basic tenet of the so-called clonal theory), due to the high amount of receptor specificities, the probability that an foreign particle entering a multicellular organism be recognized by a lymphocyte it had just encountered is very low. Since immune defences would be uneffective if an excessive amount of time was required to initiate an immune response, there is a need for rapid ways of detecting the presence of foreign material with limited specificity. Understanding the involved recognition mechanisms is a challenge of high current interest.

\subsection{Signal generation}

A general consequence of biorecognition events is the selective binding of specific molecules by cell membrane receptors and subsequent generation of intracellular signals that drive cell function. It has long been considered that this phenomenon was fully accounted for by the specificity of intermolecular recognition events. Also, signal generation was usually ascribed to two prominent mechanisms: (i) in many cases, ligand-receptor association was found to result in a change of receptor conformation with concomitant acquisition of signaling sites. G protein-coupled receptors are a general example, and more of 700 of these receptors appear to be encoded in the human genome out of about 25,000 genes (Alberts 2008). (ii) An other general mechanism of signal generation is the surface aggregation of membrane receptors following association with multivalent ligands. This aggregation may result in conformational changes, or in encounter between enzymes and substrata bound to the intracellular part of receptors. Thus, T lymphocyte activation often involves a clustering of tyrosine kinases such as p56lck, that are constitutively bound to cytoplasmic domains of so-called coreceptors. Co-receptor clustering may thus trigger the phosphorylation of tyrosines borne by the cytoplasmic chains of nearby molecules. These phosphorylated tyrosines will then become ligands for intracellular scaffolding proteins bearing cognate SH2 domains (SmithGarvin 2009). However, while the importance and frequency of aforementioned mechanisms are well established, recent reports supported the view that a number of membrane receptors might behave as force sensors and generate signals through different kinds of mechanisms. Binding of surface-attached ligands might result in force generation, thus generating conformational changes and appearance of binding sites that might nucleate signaling scaffold. Thus, recent data suggested that TCR signaling might indeed be influenced by forces (Ma 2008). Also, it was recently reported that a force of only a few piconewtons applied on molecule talin might result in the appearance of new reactive sites (delRio 2009). Thus, the effect of forces on molecules involved in recognition events is of direct functional significance.

In conclusion, most aspects of cell function are dependent on speficic interactions between biomolecules. The outcome of interaction depends on affinity, but also on association and dissociation kinetics and bond sensitivity to disruptive forces. Further, in view of the tremendous number of potential interactions occurring in the biological environment, the specificity of binding molecules is a key properties that needs to be 
rigorously evaluated. Finally, biomolecule interactions may involve soluble molecules, but also surface-attached receptors. As a consequence of this situation, it appeared during the last decades that the conventional theoretical framework developed during the last century to account for soluble phase (also called 3D) interactions was insufficient to deal with cell function. This was an incentive to develop new methods of studying interactions between surface-attached molecules (i.e. 2D interactions). These methods gave accurate information on bond formation and dissociation at the single molecule level. This exquisite sensitivity provided investigators with a direct grasp on specific aspects of molecular behaviour such as random thermal fluctuations. Data interpretation thus required a reexamination of older theoretical models. These recent developments will be rapidly sketched in the following section.

\section{Brief historical outline of recent investigations made on biomolecule recognition at the single bond level}

The purpose of this section is to give a brief account of a series of investigations essentially performed during the last two decades to analyze interactions between surfacebound molecules at the single bond level. Indeed, the kind of understanding brought by these studies proved highly relevant to biomolecule function, and this was an incentive to reexamine theoretical frameworks elaborated more than a century ago to account for the basic mechanisms of molecule association and separation (Eyring 1935, Kramers 1940, Hanggi 1990). It is hoped that this brief outline will help the reader grasp more easily the rationale of more recent work.

\subsection{Studying bond rupture at the single molecule level}

A theoretical paper authored by George Bell (Bell 1978) may be considered as a starting point to all recent work on the force sensitivity of single bonds. The purpose of Bell's paper was to find a relationship between the function of cell membrane receptors responsible for adhesive phenomena and the properties of soluble forms of these molecules. Two main points of this paper consisted (i) of separating the encounter phase of interaction, that was supposed to be different under 2D and 3D conditions, and the second phase of complex formation that was postulated to be similar in free and surface-anchored molecules, and (ii) of suggesting an simple model to account for the effect of disruptive forces on dissociation rates, leading to the so-called Bell's law:

$$
k_{o f f}(F)=k_{o f f}(0) \exp \left(F x_{\beta} / k_{B} T\right)=k_{o f f}(0) \exp \left(F / F^{0}\right)
$$

where $k_{o f f}(F)$ is the dissociation rate of a bond subjected to force $\mathrm{F}$, as shown on figure $3, x_{\beta}$ is a parameter with the dimension of a length that was interpreted as the distance between the equilibrium distance and the transition state of the ligandreceptor complex as observed on a one-dimensional energy landscape, and $k_{B}$ and $\mathrm{T}$ 


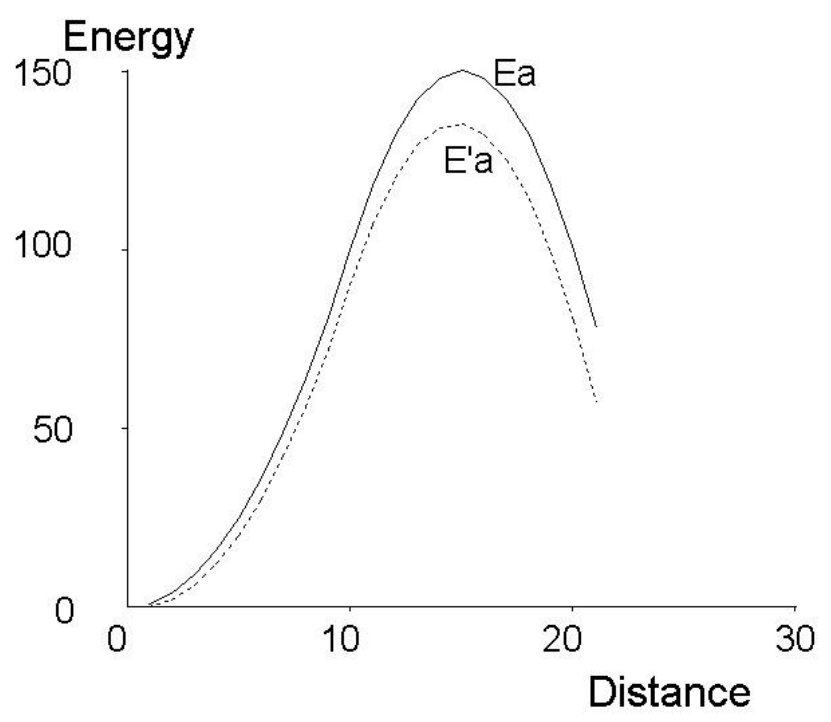

Figure 3: Bells law. Bells interpreted bond rupture as the exit of an energy well on a unidimensional energy landscape. Assuming that the frequency of particle attempts at crossing the barrier was a constant, the probability of success was estimated at $\exp \left(E a / k_{B} T\right)$, where Ea is the activation energy. The effect of a force is to lower the energy curve in proportion to the distance (broken line). (Bell 1978)

are Boltzmann's constant and the absolute temperature. F is a parameter with the dimension of a force that may be viewed as an indicator of bond mechanical strength.

This formula is now denominated as Bell's law. A theoretical justification based on Smoluchowski's equation was elaborated a few years later by E. Evans (Evans 1997). Also, while it seemed reasonable to expect that a disrupting force should reduce the lifetime of a bond, rigorous thermodynamic reasoning lead M. Dembo and colleagues (Dembo 1988) to notice that a disrupting force should reduce the affinity of a bond, but since the affinity constant is the ratio between the association and dissociation rates, it was conceivable that a pulling force might somewhat paradoxically increase bond lifetime. The authors dubbed slip bonds "normal" bonds displaying decreased lifetime in presence of forces, and catch bonds "strange" bonds displaying increased lifetime in presence of force.

Remarkably, within a few years, several complementary methods (Bongrand 1994) allowed a number of investigators to test the theoretical predictions that had recently been reported. H. Goldsmith used a moving capillary tube to monitor the rupture of doublets made between osmotically sphered red cells coated with a minimal amount of antibodies (Tha 1986) and subjected to shear flow. The normal force at separation ranged between 60 and $197 \mathrm{pN}$. Assuming that binding involved a few or even one antibody molecule, this order of magnitude was consistent with Bell's prediction. A few years later, E. Evans used a dual pipette apparatus to monitor the rupture of attachments between red cells bound by a minimal amount of antibodies (Evans 1991). He estimated at a few tens of piconewtons the rupture force and ascribed it to the uprooting of membrane molecules, a possibility already suggested by Bell (Bell 1978). 
Soon thereafter, laminar flow chambers (Figure 4) were used to monitor the formation and rupture of attachments between moving particles and surfaces coated with receptor and ligand molecules.

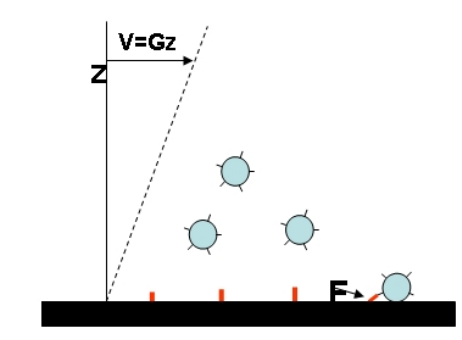

A

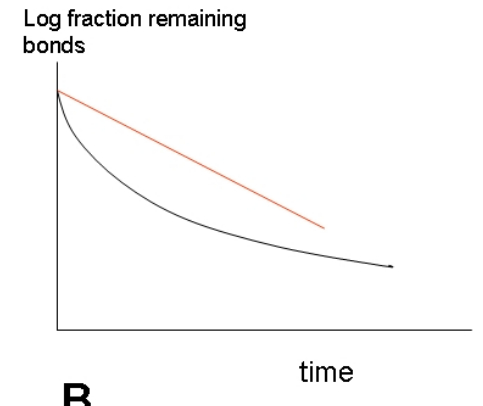

B

Figure 4: Studying molecular interactions with a flow chamber. Optimal information can be obtained by studying the motion of receptor-coated microspheres near ligand-coated surfaces in presence of a wall shear rate of a few $s^{-1}$. Using microspheres of a few $\mu m$ diameter, trajectories can be monitored with an accuracy of several tens of $\mathrm{nm}$ and time resolution of $20 \mathrm{~ms}$ with standard video equipment. The force exerted on a particle of 1.4 $\mu \mathrm{m}$ radius may be a fraction of a piconewton, and the force on the bond may be estimated at a few $\mathrm{pN}$ when the wall shear rate is on the order of several $s^{-1}$, which provides high sensitivity. The possibility to scan extensive contact areas is well suited to the use of low surface density coatings and determination of association rates. The capacity of the flow chamber to measure the kinetic and mechanical properties of weak bonds is described in a recent review (Pierres 2008).

This approach proved a highly sensitive way of observing single bond formation and dissociation, since a cell size sphere subjected to a wall shear rate on the order of a few $s^{-1}$ displays a translational velocity of a few $\mu \mathrm{m} / \mathrm{s}$ and is subjected to a distractive force on the order of a piconewton, which is sufficiently low to permit a single weak bond to maintain a particle at rest during a detectable amount of time. The lifetime of single bonds formed between E-selectin molecules and ligands borne by flowing neutrophils was estimated at about $2.4 \mathrm{~s}$ (Kaplanski 1993). During the following years, flow chambers were used to estimate Bell's $F^{0}$ coefficient for the force dependence of dissociation rates, yielding about $90 \mathrm{pN}$ for P-selectin/PSGL-1 couple (Alon 1995). However, it was soon reported that single bond rupture was more complex than predicted with Bell's law, since ligand-receptor association behaved as a multiphasic reaction (Pierres 1995, Pierres 1996). Another problem that was later emphasized was the difficulty of ensuring that single bonds were indeed observed (Zhu 2002). This difficulty may provide an explanation for the discrepancy found between different estimates of parameter $F^{0}$ (Alon 1995, Evans 2001).

Atomic force microscopy (AFM) provided another way of tackling with single bonds. Initial studies (Florin 1994, Lee 1994) were performed on the avidin/biotin interaction, that is known for its high affinity constant on the order of $10^{15} M^{-1}$. H.Gaub and colleagues reported on the rupture of association between cantilever tips deriva- 
tized with avidin and agarose beads coated with biotin or analogs. The avidin/biotin separation forces appeared as integer multiples of 160 piconewtons, which was interpreted as the strengh of a single bond (Florin 1994). As previously reviewed (Bongrand 1999), during the following years, different authors used flow chambers, atomic force microscopes, and also optical tweezers (Thoumine 2000) to measure the rupture force of a number of ligand-receptor couples. A major advance came from E. Evans' laboratory when he markedly enhanced the power of his micromechanical approach by developing the so-called biomembrane force probe (BFP) (Evans 1994, Merkel 1999): He glued a latex microbead on an erythrocyte that was used as a tunable cantilever. He used pipettes mounted on a piezoelectric system allowing computer-controlled displacement with high velocity and subnanometer accuracy. Finally, a rapid videocamera allowed excellent time resolution. This device allowed Evans to convince the scientific community that the unbinding forces commonly reported in AFM-based studies were not intrinsic parameters of a given ligand-receptor couple : indeed, even with an interaction as strong as the avidin-biotin bond, spontaneous rupture will occur in absence of force if observation is performed for a sufficiently long time (that may be centuries!). When individual bonds were subjected to a pulling force increasing at constant rate (the so-called loading rate, expressed as $\mathrm{pN} / \mathrm{s}$, the force at the moment of rupture was linearly dependent on the logarithm of the loading rate. When the loading rate was varied over an impressive range of six orders of magnitude and the rupture force was plotted versus the logarithm of the loading rate, the curve appeared as a sequence of straight lines that could be related to the localization of barriers in the energy landscape (see below). This method dubbed dynamical force spectroscopy (DFS) (Evans 2001) provided a powerful way of analyzing ligand-receptor interactions. At this stage, bond rupture might be viewed as the serial passage of a series of barriers in an one-dimensional energy landscape that could be analyzed with DFS (Evans 2001). Each barrier was crossed with a frequency that seemed to increase in presence of forces following Bell's law. Flow chambers and AFM or BFP appeared as consistent and complementary methods. Thus, while BFP gave accurate information on a notable part of energy landscapes, flow chambers operated at several wall shear rates allowed direct visualization of the random character of bond rupture (as illustrated by the distribution of bond lifetimes). The multiplicity of bound states, corresponding to the multiplicity of energy barriers, was an early finding (Pierres95). Results obtained on a same molecular model such as homotypic cadherin association with a flow chamber (Perret 2002) and BFP (Perret 2004 appeared fairly consistent. A general finding was that flow chambers were better suited to probe weak interactions or to analyze the outer part of energy landscapes of strong interactions such as avidin/biotin association (Pierres 2002a), while AFM and BFP provided more information on the inner part of these energy landscapes. While Bell's law was considered to account quite satisfactorily for many experimental models (Chen 2001) as a convenient zeroth order phenomenological theory (Dudko 2006), experimental results obtained with flow chambers (Thomas 2002, Marshall 2003) and AFM (Marshall 2003) on a bacterial model of lectin-mediated adhesion and the P-selectin/PSGL-1 interaction strongly supported the view that the catch bonds fancied by Dembo and collegues actually existed. 
This was an incentive to reexamine the theoretical framework used to analyze experimental data. Further details will be provided in the following chapters.

\subsection{Measuring bond formation at the single molecule level}

As previously emphasized (Pierres 1998), studying bond formation between surfaceattached molecules appeared more difficult than aforementioned investigations on bond dissociation for several reasons: First, while it is relatively easy to compare the frequencies of bond rupture under 2D and 3D conditions since both are expressed in the same units (i.e. second ${ }^{-1}$ ), rates of molecular association are respectively expressed in $M^{-1} s^{-1}$ and in molecule ${ }^{-1} \mu m^{2} s^{-1}$ under 3D and 2D conditions respectively (Pierres 2001, Dustin 2001). Secondly, while it is relatively simple to exert a force on a bond until it breaks, studying bond formation requires to bring two molecules into close contact, wait for a given amount of time, then exert a force to determine whether molecules are bound. However, the choice or force or waiting time is quite arbitrary and many combinations must be tried. Thirdly, while bond strengh is mainly dependent on the molecular properties of interaction sites (but see Evans 1999), the properties of linkers between molecules and surfaces may play a dominant role in binding kinetics (Pierres98a). The earliest determination of biomolecule association rate at the single bond level was performed with atomic force microscopy by P. Hinterdorfer (Hinterdorfer 1996) who studied the interaction between a mica surface coated with bovine serumalbumin (BSA) and a cantilever tip coated with anti-BSA antibodies connected through a $8 \mathrm{~nm}$ long polyethyleneglycol linker. The association rate was derived from the binding frequency, assuming free motion of the antibody site (paratope) in a half sphere. The association rate $k_{o n}$ was estimated at $5 \times 10^{4} M^{-1} s^{-1}$ which was deemed comparable to values reported on several antibody/peptide couples. Soon thereafter, a laminar flow chamber was used to measure the binding frequency of beads and planar surfaces coated with fragments of C-cadherin, an homotypic adhesion molecule (Pierres 1998). The binding frequency was estimated on the basis of computer simulations yielding a quantitative estimate of the actual interaction time between beads and surfaces, as a consequence of vertical brownian motion. The estimate of about $1.2 \times 10^{-3} \mathrm{~s}^{-1}$ for the binding frequency would yield an association rate of about $0.2 M^{-1} s^{-1}$ as estimated with a similar reasoning as that suggested by Hintertorfer. This value is much lower than an estimate of cadherin association rate obtained with atomic force microscopy (Baumgartner 2000). On the same year, a clever way of estimating association rates was reported in C. Zhu's laboratory (Chesla 1998). This consisted of generating numerous transient encounters between erythrocytes coated with immunoglobulin G ( $\operatorname{IgG}$ ) and transfected $\mathrm{CHO}$ cells expressing IgG receptors. This was achieved with two micropipettes and a piezoelectric system was used to vary the encounter time in the several second range. Binding events were revealed by transient deformation of softer erythrocytes, which allowed exquisitively sensitive count. Concomitant determination of the surface density of ligands and receptors allowed quantitative determination of the product between the conventional association rate $k_{\text {on }}$ and contact area which was estimated at $2.6 \times 10^{-7} \mu \mathrm{m}^{4} \mathrm{~s}^{-1}$. The molecular contact area was estimated at a few percent of a macroscopic contact area of $3 \mu \mathrm{m}^{2}$. More recently, Zhu et al. improved 
this method by using a more sensitive way of detecting attachments: instead of looking for a deformation of the softer cell membrane, they recorded the thermal fluctuations of the biomembrane force probe (Chen 2008, Chen 2008a). This allowed them to measure bond formation and dissociation with better than $100 \mathrm{~ms}$ resolution. During the same period of time, flow chambers went on being used to study bond formation kinetics. Careful analysis of the relationship between contact duration between surfaces and binding probability led to the intriguing finding that the probability of bond formation was not proportional to encounter duration, but rather that a minimum contact time was required to allow binding (Robert 2009). This finding might cast a doubt on the suitability of the association rate parameter to account for different experimental models. Note that this conclusion does not mean that the use of an association rate is incorrect. Only, if a binding reaction is highly multiphasic, i.e. if it involves numerous sequential reactions with a number of association rate parameters, it was found that association kinetics might be approximated with a simple law involving a single parameter. This point will be further discussed in the following section.

\section{Which parameters do we need to account for biomolecule recognition?}

The examples provided in section 2 show that a quantitative description of biomolecule recognition is required to understand how these biomolecules fulfill their function. The historical outline given in section 3 shows that a new kind of knowledge is now available concerning biomolecule interaction. On the basis of this progress, it is now warranted to reexamine the suitability of older parameters used to investigate molecular interactions. It is important to notice that there is a certain degree of freedom in the choice of basic parameters. As an example, either forces or energies might be chosen as primitive parameters for developing theoretical mechanics. However, it is important to understand that an improper choice might lead to conceptual limitation and seriously hamper a quantitative interpretation of experimental data. This point may be illustrated with the following two examples.

(i) When the force-induced rupture of molecular bonds began being studied at the single molecule level, a natural parameter might be the rupture frequency $k_{o f f}$, as was naturally chosen when laminar flow chambers were used as experimental devices (Kaplanski 1993, Alon 1995, Pierres 1996). However, the unbinding force was chosen by investigators using atomic force microscopy (Florin 1994, Hinterdorfer 1996, Baumgartner 2000). Theoretical (Evans 1997) and experimental (Merkel 1999) advances were needed to show that unbinding forces were not intrinsic parameters and were strongly dependent on loading rates. This new understanding may be considered as the starting point for dynamic force spectroscopy (Evans 1997, Merkel 1999).

(ii) A common theoretical procedure initiated by H. Eyring consisted of modeling bond formation and dissociation as consequences of time dependent evolution of a single coordinate in a unidimensional energy landscape, which was interpreted as a valley in a multidimensional hypersurface (Eyring 1935). Deeper analysis was needed to understand that the choice of a reaction coordinate is by no means straightforward, 
since an improperly chosen coordinate cannot provide an "intrinsic" description of a system if it is dependent on the system history (Best 2005).

Thus, a good set of interaction parameters must satisfy the following criteria: (a) it must be sufficiently exhaustive to predict biomolecule behaviour when numerical values of parameters are known, (b) This should be liable to experimental determination, (c) this must be sufficiently "intrinsic" to be independent of a particular experimental setup used for experimental study, (d) it should be feasible to relate each parameter to molecular structure.

Commonly used interaction parameters (or coordinates) will now be rapidly considered.

\subsection{The affinity constant}

As previously acknowledged (Williams 1991), the concept of affinity still dominated most thinking about complex biological reactions only two decades ago. Starting from the standard equation:

$$
A+B \rightleftarrows(A B) ; K_{a}=\frac{[A B]}{[A][B]}
$$

where $\mathrm{A}$ and $\mathrm{B}$ are a ligand and a receptor molecule, $[\mathrm{A}],[\mathrm{B}]$ and $[\mathrm{AB}]$ are respectively the molar concentrations of isolated molecules $\mathrm{A}$ and $\mathrm{B}$ and of the molecular complex $\mathrm{AB}$, and $K_{a}$ is the affinity constant, we can in principle calculate the amount of complex if we know the total amounts of molecules A and B. Further, determining the affinity constant between soluble receptors and ligands may be easily achieved with powerful and widely available methods such as are based on optical biosensors (Schuck 1997) (some caution is however warranted (Rich 2006). Finally, the thermodynamic relationship :

$$
K_{a}=\exp \left(-\Delta G^{0} / R T\right)
$$

allows us to relate the affinity constant to the free enthalpy of reaction under standard conditions (see standard treatises or (Bongrand 1999) for more details). However, there are two problems with this formalism:

Firstly, while equation 2 is useful under equilibrium conditions, life works out of equilibrium. As an example, the affinity constant may conveniently account for the amount of occuped receptors on the cell membrane in a stable environment, but it is certainly insufficient to account for the evolution of rapid signaling cascades.

Secondly, while equation 2 can be used to deal with two soluble reactants, or a cell receptor interacting with soluble ligands, it cannot account for interactions between surface-attached molecules. A major problem is related to the reaction entropy. As emphasized by Page and Jencks, the standard free enthalpy $\Delta G^{0}$ is the sum of an "intrinsic term" that represents the intrinsic binding energy and a connecting term that represents the loss of entropy generated by complex formation (Page 1971, Jencks 1981). The problem is that both terms are of comparable order of magnitude and they may be quite different when interacting molecules are bound to surfaces, which may dramatically restrict their motion and number of degrees of freedom.

Reasoning with kinetic parameters instead of affinity constants may suffice to deal with out-of-equilibrium processes. As was emphasized, dealing with surface- attached 
molecules will result in the replacement of two numbers, the reaction on-rate and off rate, with two functions, namely $k_{o f f}(F)$, i.e. the dissociation rate as a function of applied force, and $k_{o n}(d)$, i.e. the association frequency of two molecules maintained at a fixed distance d (Pierres 1996b). The suitability of these function will be considered below. Unfortunately, this additional complexity remains insufficient to deal with all situations of biological interest.

\subsection{Kinetic constants : the on-rate and the off-rate}

The kinetic description of molecular interaction may seem more intuitive than the thermodynamic description. It makes use of two parameters as follows:

$$
A+B \underset{k_{\text {off }}}{\stackrel{k_{o n}}{\rightleftarrows}} A B \quad ; \quad d[A B] / d t=k_{\text {on }}[A][B]-k_{o f f}[A B]
$$

Accounting for the kinetics of molecular interactions certainly contributed a major advance to the study of many biological phenomena. Thus, kinetics certainly plays a major role in determining the respective role of selectin and integrin adhesion receptors in leukocyte interaction with blood vessels. When a cell briefly encounters a foreign surface, only kinetic information can tell us whether contact will be durable enough to allow bond formation provided suitable receptors and ligands are expressed on surfaces. The remarkable treadmilling phenomenon (Alberts 2008) reported on cell cytoskeletal elements is understandable only on the basis of kinetic data: actin microfilaments are oriented, and while the thermodynamics of monomer association/dissociation are similar on both ends, there is a tenfold difference between kinetic constants. Also, signaling cascades generated by membrane receptors require the rapid formation of multimolecular scaffolds that are strongly influenced by interaction kinetics as well as molecular localization. Finally, recent methodological advances such as the use of surface plasmon resonance technology allowed rapid increase of available data on the kinetics of a number of ligand-receptor couples (Schuck 1997) and experimental progress was an incentive to consider more thoroughly the significance of kinetic rates. For the sake of clarity, bond formation and dissociation will be considered separately. Bond dissociation will be first considered in view of its greater simplicity and historical order.

\subsubsection{The force-dependent dissociation rates}

Since the principles of bond dissociation with AFM and BFP and theoretical interpretations are described with much detail in chapters 2 and 3, only some key points will be mentioned.

- First, dissociation rates are highly relevant to important experimental situations: as mentioned above, the outcome of interactions between a ligand and a receptor is certainly dependent on interaction lifetime. Prominent examples are (i) cell adhesion, since an essential factor of adhesion efficiency is the capacity of a single bond to maintain a cell in contact with an adhesive surface until a second bond occurred. This is the critical step to the formation of a firm adhesion that will be maintained by hundreds or thousands of bonds (Bongrand 1984, Pierres 2000). and (ii) signaling, since 
in some cases exemplified by the TCR the duration of interaction will shape the cell response (McKeithan 1995). It is therefore of obvious interest to determine dissociation rates. As illustrated by the many studies on leukocyte-endothelium interaction, the force dependence of interaction plays a dominant role in some situations.

- Second, as described above and in other chapters, some techniques allow experimental determination of $k_{o f f}(F)$, i.e. the rupture frequency of a given bond in presence of a disruptive force $\mathrm{F}$. This may be achieved with a flow chamber which yields direct determination of $k_{o f f}(F)$ (Pierres 2008b) or with AFM or BFP, since theoretical models allow us to relate constant-force binding frequencies and unbinding forces measured at constant pulling speed (Dudko 2008, Freund 2009). An important point is that the force-free dissociation rate and mechanical resistance may behave as different parameters: thus, when the ligand CD34 of L-selectin was subjected to mild periodate oxidation, the force free dissociation rate $k_{o f f}(0)$ was not substantially altered, in contrast with dissociation rates measured in presence of disruptive forces, as evidenced with a flow chamber (Puri 98). This example supports the use of considering the force dependence of dissociation rates.

- Third, an important question is to know whether $k_{\text {off }}(F)$ may be viewed as an intrinsic property of a given ligand- receptor complex AB. While a positive answer might have seen obvious a few years ago, two recent papers (Pincet 2005, Marshall 2005) reflected the feeling that bond lifetime and dissociation rates were not intrinsic parameters since they depended on the history of studied complexes. This apparent paradox is indeed a consequence of a clear approximation in our language: it is only an approximation to refer to a complex $\mathrm{AB}$, since it is well known that $\mathrm{AB}$ may span a number of states that appear as local minima in a muldidimensional energy landscape or even in a one-dimensional reaction path (Zwanzig 1988, Pierres 1995, Merkel 1999). Therefore, if the amount of time required to reach equilibrium is higher than the period of time between complex formation and dissociation rate determination, measured parameters will depend on thie initial state of the molecular complex and on the time allowed for equilibration between different substates before beginning measurements. An additional point is that the dissociation probability of a molecular complex subjected to a time-dependent disruptive force is dependent on the history of force application (Marshall 2005, Walton 2008) and possibly, as suggested by molecular dynamics simulation, on the precise location of atoms at the moment of force application (Walton 2008).

There are other properties that hamper the universality of function $k_{o f f}(F)$. Firstly, dissociation may depend not only on the intensity of a disruptive force but also on its direction (Astrof 2006, Zhu 2008). This may be important if free rotation is not allowed between binding molecules and surfaces. Secondly, dissociation is not only dependent on the properties of binding sites but also on linker molecules connecting these sites to surfaces (Evans 1999, Walton 2008).

Fourth, several authors developed theoretical models to relate dissociation frequencies under constant load or loading rates to the location and depth of energy landscapes. The next step would be to relate these geometric and energy parameters to structural properties of binding molecules. This point will be rapidly considered in the next section. 
In conclusion, while it might appear for some years that equation 1 provided a tractable way of describing the force dependence of molecular bonds (Chen 2001), more recent work showed that i) $k_{o f f}(F)$ was often more complicated than suggested by Bell's law due to the existence of multiple barriers and possibly multiple dissociation pathways, as suggested to interpret catch bond behaviour (Pereverzev 2005), and ii) a function such as $k_{o f f}(F)$ may not exist, even with a more complicated form than equation 1, due to the effect of history and dependence on the properties of linker molecules.

\subsubsection{Distance-dependent association rates}

The importance and significance of association rates (i.e. $k_{\text {on }}$ parameter) will now be discussed.

First, there are many important examples supporting the prominent biological importance of association rates. As indicated above, the efficiency of selectin molecules was ascribed to their capacity to tether rapidly flowing leukocytes to endothelial cells, which required a particularly high association rate. Also, experimental data supported the view that the association rate of antibodies progressively increased during the socalled maturation of immune responses, a finding that was intepreted as a premium on the capacity to bind target rapidly (Foote 1991). Finally, the cell capacity to probe its environment is dependent on the capacity of membrane receptors to bind to their ligand during a transient approach of a receptor-bearing membrane protrusion towards a ligand-bearing surface. In all these case, it seems that the efficiency of bond formation should be calculatable if we knew a function $k_{o n}(d)$ defined as the frequency (per unit of time) of bond formation between a ligand and a receptor molecules located at distance $d$. Such a function would include sufficient information to account for interactions between soluble molecules (i.e. 3D conditions) and surface-attached molecules (i.e. $2 \mathrm{D}$ conditions). Unfortunately, the determination and even the very definition of such a function are fraught with difficulties for at least two complementary reasons.

i) if the association between molecules $\mathrm{A}$ and $\mathrm{B}$ is a multiphasic reaction involving a high number of interaction states, the discrimination between free and bound states may be somewhat arbitrary. Indeed, if bond formations is not an all-or-none phenomenon but requires a progressive strengthening, it it not obvious to chose a threshold to discriminate between free and bound states. Thus, while the streptavidinbiotin interaction might have been considered as strong enough to allow easy detection of bound states, several investigators reported on the time-dependent maturation of this interaction (Pincet 2005) and existence of a number of weak association states (Pierres 2002). Indeed, if the number of intermediate states is high, the concept of association constant becomes meaningless. This point was recently demonstrated in a quantitative study made on the binding efficiency of antibody-coated microspheres encountering antigen-bearing surfaces in a laminar flow chamber (Robert 2009): the probability of bond formation scaled as a power of encounter duration that was significantly higher than 1 , and under a number of conditions this probability varied as $\operatorname{erf} c\left[\left(t / t_{0}\right)^{1 / 2}\right]$, where $t$ was the contact time and $t_{0}$ was a constant on the order of $10 \mathrm{~ms}$. It was further shown that this formula could be derived from a simple model 
where the reaction landscape was modeled as an unidimensional curve with a rugged segment (Figure 5). It may be useful to emphasize that this problem could not have been detected in studies of 3D interactions, since in this case the encounter time is determined by the laws of diffusion and is not expected to display substantial variations between different experimental setups.

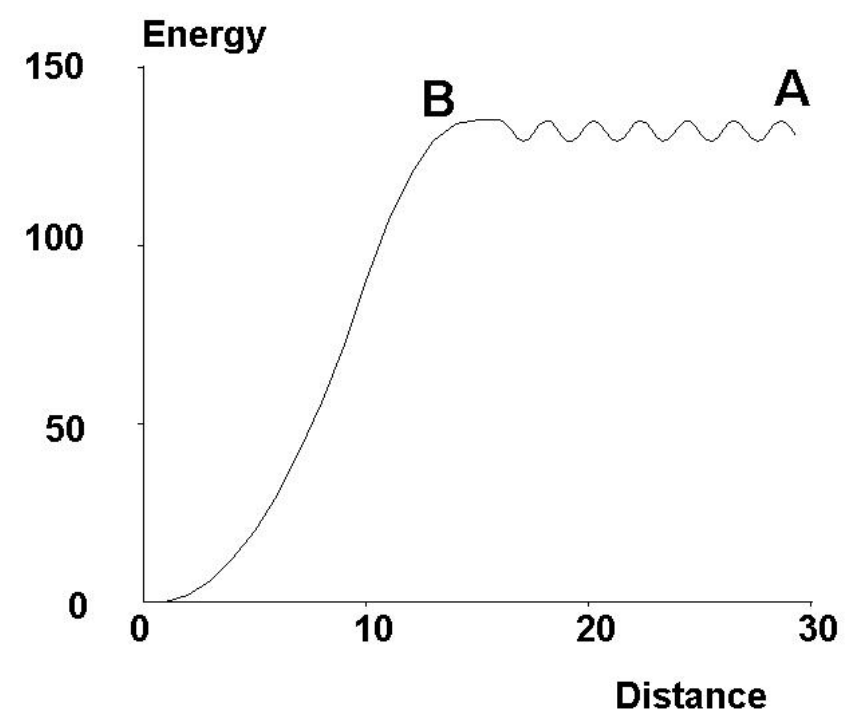

Figure 5: Model of bond formation. Bond formation is modeled as a passage through a rough segment of an energy landscape, represented as passing from $\mathbf{A}$ to $\mathbf{B}$. This was found to match experimental findings obtained with a flow chamber (Robert09).

ii) Atomic force microscopy and micropipette-based methods, that met with impressive success in analyzing bond rupture, may be less well suited to the study of bond formation because they do not allow easy control of contact duration, since contact is usually difficult to detect. Also, the contact area is often difficult to estimate since it is difficult to observe (Chesla 1998) and it may be markedly altered by forces exerted by the apparatus to induce molecular contact (Thoumine 2000). Finally, while hundreds or thousands of approach/retraction cycles can be performed on a given contact area, it is more difficult to sample extensive areas, which may be useful if low ligand and receptor densities are used in order to ensure that binding events are representative of single bond formation and dissociation.

Further, when ligands and receptors are attached to surfaces, association rates are less "intrinsic" parameters than dissociation rates because bond formation is highly dependent on the properties of linker molecules (Jeppesen 2001). Indeed, if molecules are rigid, association will be impossible if ligands and receptors are not suitably oriented to allow proper match between interacting areas. In contrast, association will be strongly enhanced if ligands and receptors are forced against each other with binding configuration. Also, the microtopology of surfaces bearing ligands and receptors may strongly influence association rates. As an example, the association rate between capsules bearing immunoglobulins and immunoglobulin receptors displayed 50-fold decrease when a smooth erythrocyte was replaced with a rough nucleated cell (Williams01). 
In conclusion, The new kind of information that was recently obtained on biomolecule interactions by studying single bond formation and dissociation in presence of forces is directly relevant to a number of important biological processes. However, connecting this information to structural data still requires significant theoretical and experimental progress. In addition, accounting for biological processes still requires to consider other less well defined parameters than $k_{o f f}$ and $k_{o n}$. Thus, we shall briefly discuss the frequently used concepts of avidity and specificity.

\subsection{Avidity of biomolecule interactions: an incompletely de- fined parameter}

While aforementioned development might convey the view that ligand-receptor interaction are liable to rigorous quantification, it has long been recognized that the affinity constant or association rates did not fully account for biological phenomena. Antigen recognition by antibodies provides a suitable example in view of the huge diversity of interactions and number of applications in hospital and research laboratory. As written in a standard treatise several decades ago (Glynn 1977) In the literature, affinity and avidity commonly are used synonymously ... However, it is now accepted that the term affinity is a thermodynamic expression ... Avidity also involves other contributing factors such as antibody valence, antigen valence. A similar opinion remains in use today (Murphy 2008) ... The total binding strength of a molecule with more than one binding site is called the avidity. Thus, although it is accepted that avidity is not defined as accurately as affinity, a general concept is that this may be related to the capacity of forming multivalent associations. Indeed, many situations suggest that a most common way of forming strong associations involves the formation of multiple bonds. The following examples are intended to support the importance of the concept of avidity and the complexity associated to the multivalency of molecular interactions.

Many biological interactions need to be multivalent. There are many examples suggesting that a single noncovalent interaction between a ligand and a receptor may be too transient to be significant. Cell adhesion is driven by a number of membrane receptors that often require multivalent interactions. Cadherins, that are thought to play a dominant role in the stability of epithelia, are an important example. The importance of lateral clustering was very elegantly demonstrated (Yap 1997) by studying the adhesion of cells expressing engineered cadherins that could be oligomerized at will by bridging the intracellular domains with a drug. Similarly, integrins play a prominent role in cell adhesion to extracellular matrix components. It has long been known that cell surface integrins are often in an inactive state and events including clustering or conformational changes are required to enable these integrins to bind their ligands. Some recent examples clearly demonstrated that clustering integrins could directly enhance the binding to multivalent, not monovalent ligands without any affinity change (Bunch 2010). As another example, ICAM-1, a ligand of integrin LFA-1, was reported to bind to immobilized LFA-1 with high avidity (dissociation constant was $8 \mathrm{nM}$ ) after dimerization, while no measurable interaction was observed with monomeric ICAM-1 (Miller 1995). It was further checked that ICAM-1 monomer expressed a complete LFA-1 binding surface (Jun01) 
It is difficult to relate the properties of divalent and monovalent interactions. As emphasized above, it is because of this difficulty that single molecule studies revolutionized our understanding of biomolecule interactions. This difficulty is due to several reasons. Firstly, the rupture frequency of multivalent attachments may be drastically decreased by the possibility of rebinding events. Indeed, while a monovalent attachment is expected to break spontaneously as a consequence of thermal fluctuations, a multivalent attachment may need an external force for rupture if rebinding occurs (Seifert 2001). Also, the force sensitivity of multivalent attachments is strongly dependent on force sharing between different bonds, and unbinding forces may follow a number of different laws depending on forces and bond arrangement (Seifert 2000, Sulchek 2005, Tang 2007).

In conclusion While single molecule studies essentially provided an accurate description of the interaction between binding sites exposed by biomolecules, we need to better understand the requirement for multivalent association. Clearly, multivalency is dependent on the topographical relationship between different binding sites and molecular flexibility. When interactions involve surface-attached molecules, other additional factors are important, including static and dynamic length and flexibility of linkers between surfaces and binding sites, as well as rugosity of the surface region surrounding molecules, and lateral mobility of molecules. These points will be briefly listed in a later section.

\subsection{Specificity of biomolecule interactions: an essential prop- erty that is difficult to define accurately}

Obviously, biomolecules must bind specifically to adequate targets in order to fulfil their task. Specificity seems easy to define qualitatively : a ligand-receptor interaction is the more specific as the interaction between the same receptor and a "slightly" different ligand is "weaker". However, there is no general way of defining the similarity of two molecules or the strength of an interaction.

First, two molecules may differ according to their shape (e.g as mentioned above for ortho- or para-dinitrophenol), their electric charge, their hydrogen bonding capacity or their hydrophobicity. As indicated in the next section, all these properties are involved in biomolecule recognition, but their relative importance may be different in varying situations. The similarity (or dissimilarity) between two molecules is not an absolute quantitative concept. This arbitrariness was indeed pointed out many years ago (Janin 1996).

Second, an interaction may be considered as "weaker" than another one if it occurs less often under physiological conditions. Thus, the affinity constant may be the dominant parameter if we are interested in the proportion of receptor molecules that are occupied by their ligand at equlilibrium, e.g. the number of insulin receptors at a given moment. However, if we are interested in the detection of an immobilized ligand on a surface dynamically explored by a cell protrusion, the kinetic rate of bond formation may be more important. Finally, if we are interested in the specificity of cell tethering on a surface, the interaction strengh may be the dominant parameter. 
Interestingly, these parameters are not necessarily correlated. Thus, when mutant streptavidin molecules were made to bind biotin, the rupture forces were different, but they were correlated to the thermodynamic enthalpy rather than free energy of reaction (Chilkoti 1995), which is tightly related to the affinity constant, as recalled in eq. 2. Also, as mentioned above, the zero-force dissociation rate may not be correlated to the force resistance as represented with Bell's distance parameter (Puri 1998, Pierres 2006)

Third, the difficulty of defining specificity is further illustrated by so-called promiscuous receptors that may bind specifically a number of very different ligands, while a slight alteration of a given ligand may abolish the interaction. As an example, a monoclonal antibody was reported to bind specifically 2,4 dinitrophenol $\left(K_{d}=20 n M\right)$, with a negligible affinity for the close analogs 2-nitrophenol and 2-nitro-4-iodophenol $\left(K_{d}>100 \mu M\right)$, but which also bound unrelated compounds such as furazolidone with high affinity $\left(K_{d}=1.2 \mu M\right)$ (James03)

In conclusion, while it is recognized that both affinity and specificity are essential properties of ligand-receptor interactions (Janin 1996, Wang 2006), the latter may remain difficult to define unambiguously. Specificity cannot be considered as an intrinsic parameter. A receptor may be considered as specific for its ligand if it does not interact with other molecules that it is liable to encounter under biologically relevant situations. The significance of interaction specificity will be discussed more precisely in a further section devoted to the structural basis of biomolecule interactions.

\subsection{Ligand-receptor interactions are influenced by parameters that are extrinsic to both ligand and receptor molecules}

In addition to the parameters we have just mentioned, it is important to recall that molecular associations occurring in the biological milieu may be deeply influenced by a number of external parameters that may obscure the intrinsic properties of interacting sites. Receptor-mediated cell adhesion provides many examples as shown below. We shall give selected examples to illustrate this point.

Presence of repellers on receptor-bearing surfaces. It is well known that the surfaces of living cells are coated with a carbohydrate-rich layer with a thickness of several tens of nanometers or more, called the glycocalyx or pericellular matrix. Much experimental evidence supports the view that i) The glycocalyx may substantially impair the receptor capacity to bind to ligands, particularly during short encounters as occur in a laminar flow chamber (Sabri 1995, Patel 1995). ii) Under some circumstances, cells may increase their receptor capacity by rapid removal of glycocalyx components, thus increasing the accessibility of membrane receptors (Sabri 2000). This inhibitory effect of the pericellular matrix is an example of so-called steric repulsion (Pierres 2000).

Lateral mobility of binding molecules It seems obvious that the probability of encounter between surface-attached binding molecules may be strongly enhanced if molecules can move freely on surfaces. This has long been demonstrated experimentally. As an example, when cells bearing CD2 surface molecules were micromanipulated into contact with surfaces coated with CD58, a ligand of CD2, either in immobilized 
form or freely diffusing in a supported lipid bilayer, adhesion efficiency was strongly increased when ligand molecules were mobile, and this effect was more apparent when the ligand density was decreased (Chan91). As another example, the adhesive efficiency of cell surface integrins was reported to increase in parallel with lateral mobility, as measured with enhanced video microscopy and single particle tracking (Kucik96).

Localization of binding molecules on surfaces. As previously indicated, surface roughness may strongly decrease the accessibility of surface receptors (Williams 2001). It is understandable that this phenomenon might depend on the localization of binding molecules as suggested by some experimental evidence. Thus, the capacity of selectin molecules to mediate binding of rapidly flowing leukocytes to the vessel walls was found to require the localization of these selectins on the tip of cell surface protrusions. Indeed, when this localization was prevented by changing the transmembrane domain of adhesion molecules, the dynamic binding capacity was abolished although binding sites were intact (vonAndrian 1995, Buscher 2010).

Interactions between soluble biomolecules are also environment-sensitive. Recently, the kinetics of DNA hybridation was studied in living cells transfected with FRET-labeled double strand DNA (Schoen 2009) : different kinetics were observed within cells and in the extracellular milieu, and differences were dependent on the length of strands. Further, the authors did not observe any direct effect of molecular crowding in vitro. Other authors concluded on the basis of experiments and computer simulation that the molecular crowding observed in the cell interior might change protein conformation (Homouz 2008).

In conclusion, the function of biomolecules involved in recognition events is dependent on a wide spectrum of parameters, that are not all determined by the structure of binding sites or event of linker parts of binding molecules. It is certainly warranted to devote much attention to all these parameters in the forthcoming years.

\section{Relationship between biomolecule structure and recognition events}

As shown in the previous sections, the efficiency and selectivity of biomolecule interactions are dependent on a number of thermodynamic, kinetic and mechanical parameters that can be determined experimentally with exquisite sensitivity, using a number of recently developed methodologies. These advances increase our need for a theoretical framework allowing us to relate these quantitative binding parameters to structural properties. In addition to a mere intellectual appeal, such a framework would be useful i) to help us integrate a daunting amount of available data, ii) to take advantage of increasingly available structural data to predict the interaction behaviour of important molecules, and iii) to facilitate the rational design of molecules with desired interaction properties, e.g. to act as drugs. In the present section, three points related to this goal will be considered: i) the main intermolecular forces responsible for biomolecule recognition will be rapidly listed. ii) We shall describe some experiments aimed at determining which forces are involved in the interactions between molecules of known structure. iii) Lastly, we shall rapidly discuss the interest of computer simulations as 
a practical way of increasing our understanding of biomolecule interactions. All these points were sketched in a previous review (Bongrand 1999).

\subsection{Intermolecular forces in the biological milieu}

An obvious prerequisite to relate biomolecule structure and recognition events is to know the basic physical forces involved in these interactions. As written more than half a century ago in a celebrated treatise on quantum chemistry (Eyring 1944) In so far as quantum mechanics is correct, chemical questions are problems in applied mathematics. Unfortunately, the complexity of proteins makes it totally unfeasible to derive biomolecule behaviour from basic equations and there is a need for some clear guidelines to help us build an intuitive view of molecular interactions. Thus, many authors looked for a classification of intermolecular forces and it must be understood that there has to be some arbitrariness is such an endeavour. Indeed, van Oss (van Oss, 1991) found 17 so-called "primary interactions" after compiling a number of contemporary papers. Here, we shall only mention some characteristic features of four sets of forces and we refer the reader to a standard treatise (Israelachvili 1991) or a review or ours (Bongrand 1999) for more details.

\subsubsection{Electrostatic forces}

Electrostatic forces clearly constitute the basis for noncovalent interactions between biomolecules. In vacuum, the free energy of interaction between two charges q and q' separated by a distance $r$ is simply:

$$
F=\frac{q \times q^{\prime}}{4 \pi \epsilon_{0} r}
$$

where $\epsilon_{0}$ is the dielectric constant of vacuum. Since we are interested in orders of magnitude, it is interesting to indicate that the interaction energy of two unit charges of $1.6 \times 10^{-19}$ Coulomb separated by a distance $r$ expressed in $\AA$ is about $552 k_{B} T / r$, where $k_{B}$ is Boltzmann's constant and $\mathrm{T}$ is the temperature. This means that the interaction energy of two unit charges in contact in vacuum would be more than twentyfold higher than any standard ligand-receptor interaction such as were described in the first section of this review.

However, in a material medium, the electric field generated by any charge results in a polarization and orientation of surrounding molecules, which generates a counterfield tending to decrease the total electric field. As a consequence, parameter $\epsilon_{0}$ of equation 5 must be multiplied by the relative dielectric constant $\epsilon_{r}$ which is close to 78 in water. This high value is a consequence of two specific features of water : first, water molecules have a permanent dipole moment. Second, the dipole moments are highly correlated due to hydrogen bonds. This results in a particularly efficient alignment along surrounding electric fields (Eisenberg 1969). Unfortunately, it is difficult to use equation 5 together with the experimental value of $\epsilon_{r}$ to estimate the energy of electrostatic interactions in water when charges are at short distance, since i) water can no longer be approximated as a continuous medium, ii) water molecules may be 
expelled by steric effects, iii) water structure is expected to be altered near surfaces. iv) The relative dielectric constant may decrease in presence of a very high electric field, a phenomenon known as dielectric saturation. The effective dielectric constant may thus be much lower than 78 .

In addition to the charge screening by water molecules, the interaction between two fixed charges is decreased by surrounding ions : indeed, according to Boltzmann's law, it is expected that free ions will get concentrated around ions of opposite charge. This phenomenon is well accounted for by Poisson-Boltzmann's equation:

$$
\Delta V=\left[\left(\rho+\sum_{i} c_{i} q_{i} \exp \left(-q_{i} V / k_{B} T\right)\right] / \epsilon\right.
$$

where $\rho$ is the volume density of charges other than soluble ions, $c_{i}$ and $q_{i}$ are the concentration and charge of ionic species $i$. A notable simplification is achieved if the terms $q_{i} V / k_{B} T$ are low enough to use a linearized form of this equation. The interaction energy between two charges q and q' at distance r may then be written as :

$$
F=\frac{q q^{\prime} \exp (-\kappa r)}{4 \pi \epsilon r}
$$

In a 1:1 electolyte solution such as physiological saline, parameter $\kappa$ is equal to:

$$
\kappa=\left[\frac{2 c q^{2}}{k_{B} T \epsilon}\right]^{1 / 2}
$$

In a $0.15 \mathrm{M} \mathrm{NaCl}$ solution, the Debye - Hückel length $1 / \kappa$ is about $8 \dot{A}$ at room temperature. Unfortunately, the linear approximation is not always fully valid in the biological milieu. The increase of computer power led to a revival of interest in the classical equations of electrostatic and numerical solution of Poisson-Boltzmann's equations allowed investigators to build maps of the electrostatic potential of protein surfaces, based on the surface distributions of charged aminoacids such as glu, asp, lys or arg. This allowed clear visualization of active sites, thus demonstrating the importance of electrostatic interactions in biomolecule recognition (Honig 1995). The effective interaction energy between two opposite charges such as a $\mathrm{COO}^{-}$and a $\mathrm{NH}_{3}^{+}$group in a protein-protein interface was estimated at about $1.6 k_{B} T$ in an experimental study made on the high affinity interaction between thrombin and hirudin, which involves 4 electrostatic bonds (Stone 1989).

\subsubsection{The hydrogen bond}

In addition to charged ionic groups born by acidic or basic aminoacids such as asp, glu, lys or arg, the surface of any protein bears local charges resulting from the differential distribution of atomic nuclei and electronic charges. These charges are expected to generate attractive or repulsive interactions between approaching protein surfaces. A prominent example is provided by hydrogen bonds: As a consequence of the high electronegativity of atoms such as oxygen or nitrogen, the electronic cloud involved in a bond such as $\mathrm{O}-\mathrm{H}$ or $\mathrm{N}-\mathrm{H}$ is asymmetric, resulting in a net negative charge on 
oxygen or nitrogen and a net positive charge on the proton $\mathrm{H}$. As a consequence, an atom with a net negative charge such as oxygen, will be attracted by $\mathrm{H}$ atoms bearing a net positive charge. While this simple mechanism may be considered as the basis of the hydrogen bond, it must be emphasized that this is only an approximation, and a quantum mechanical approach is required to achieve a more accurate description of this interaction. A notable consequence is that the hydrogen bond is dependent on the orientation of interaction molecules, not only on the distance between positive and negative sites, and there is still an interest in quantitative modeling of this interaction (Choi 2010). Hydrogen bonds such as O...H-O play a major role in protein or nucleic acid organization. Also, they are thought to account for the highly particular structure of water (Eisenberg 1969): the $\mathrm{H}_{2} 0$ molecule may be viewed as a tetrahedron with two positive and two negative charges on the four vertices, which may allow extensive clustering of these molecules. This extensive hydrogen bonding capacity of water is responsible for its high boiling point and dielectric constant.

As a consequence, protein-protein association often results in the replacement of protein-solvent hydrogen bonds with protein-protein hydrogen bonds, which makes it difficult to predict the total contribution of hydrogen bonds to biomolecule interactions. While the energy of a typical hydrogen bond may be on the order of about $8 k_{B} T$, the contribution of an hydrogen bond to a protein-protein interaction may not be higher than $k_{B} T$ (Connelly 1994).

\subsubsection{Different timescale: electrodynamic interactions}

In addition to the aforementioned rather static view of electrical forces between fixed charges in a biological environment, other interactions are dependent on different timescales and deserve a separate treatment. The basis is the presence of a dipole of moment $\vec{p}$ that may be permanent or induced by the presence of a surrounding electric field $\vec{E}$ according to equation 9 :

$$
\vec{p}=\alpha \vec{E}
$$

where $\alpha$ is called the polarizability. Further, the interaction energy between a dipole and an electric field is simply the scalar product $-\vec{p} \cdot \vec{E}$, and the electric field generated at point $\vec{r}$ (starting from the dipole) is:

$$
\vec{E}=(1 / 4 \pi \epsilon) \operatorname{grad}\left(\vec{p} \cdot \vec{r} / r^{3}\right)
$$

These equations are the basis of two interactions:

The so-called Keesom interaction term represents the interaction between two freely rotating dipoles $p_{1}$ and $p_{2}$. Following Boltzmann's law, the relative orientations leading to a decreased free energy are favoured. After integrating over all orientations and weighting with Boltzmann's factor, one obtains:

$$
F_{K}=-\left(p_{1}^{2} p_{2}^{2} / 24 \pi^{2} \epsilon^{2} k_{B} T\right) / r^{6}
$$

For two water molecules, the numerator is about 4,300 $k_{B} T$ when $\mathrm{r}$ is expressed in $\AA$. The timescale of molecular rotations ranges between picoseconds and nanoseconds 
depending on molecule size. The effective dielectric constant is expected to decrease according to rotation velocity.

The interaction between a dipole and polarizable molecule (see equation 9) is called Debye interaction. While this is dependent on temperature, there is a nonzero high temperature limit since the net interaction between a dipole and a polarizable molecule is attractive whatever the dipole orientation. This limit is proportional to $r^{-6}$, similarly to Keesom interaction.

Finally, it is known that even in absence of permanent dipole moments two polarizable molecules located at distance $\mathrm{r}$ exert a mutual attraction. This is called dispersion or London force, since the first calculation of the dispersion interaction between hydrogen atoms was calculated by London (1930). This is proportional to $r^{-6}$. The numerical coefficient for two water molecules is about $740 k_{B} T$ when $\mathrm{r}$ is expressed in $\AA$.

Thus, there is some theoretical support for the concept that two freely interacting molecular groups will exert a mutual attraction with an energy proportional to $r^{-6}$. This is often denominated as van der Waals attraction. Further, it is well known that in addition a short-distance repulsion will prevent a collapse of molecule pairs. This repulsion was sometimes called Born repulsion. It displays very rapid variation with distance, which led to represent it empirically either as a step function (this is the simplest "hard wall" model), or as a $r^{-12}$ function, leading to the empirical 6-12 or Lennard Jones potential:

$$
F_{L J}=-4 \epsilon\left[\left(\sigma / r^{6}\right)-\left(\sigma / r^{12}\right)\right]
$$

\subsubsection{Using the formalism of surface physical-chemistry. Hydrophobic bonds.}

The sharp energy distance relationship illustrated by equation 12 is an incentive to view molecular interactions as contact forces occurring at the interface between rigid bodies. Clearly, this is not a rigorous model. A major problem is that molecular interactions are not fully additive (Margenau 1969, Yang 2003). However, this view is simple enough to be felt useful, at least as a first approximation leading us to describe biomolecule interactions as a set of contact interactions between contacting groups. This concept provides a convenient way of accounting for the so-called hydrophobic bond. The formalism of surface chemistry provides a convenient framework to discuss this point: As already indicated, the free energy of interaction between two molecules numbered 1 and 2 embedded in a medium 3 results from a balance between bond formation and bond rupture as follows:

$$
F_{12}^{(3)}=F_{12}-F_{13}-F_{23}+F_{33}
$$

since the formation of an interface between surfaces 1 and 2 (12) results in the destruction of interfaces (13) and (23) representing the region of biomolecules surfaces that will be involved in interaction, and release of solvent molecules that will exert a mutual attraction. Now, a convenient classification consists of discriminating between dispersion forces and polar interactions generated by charged groups, permanent dipoles and 
hydrogen bonds. Considering water, the surface tension is about $72 \mathrm{~mJ} / \mathrm{m}^{2}$, as compared to about $20 \mathrm{~mJ} / \mathrm{m}^{2}$ for many apolar substances. In a first approximation, it may be assumed that the difference of $52 \mathrm{~mJ} / \mathrm{m}^{2}$ represents polar interactions and that apolar components do not display important variations in different aminoacids. According to equation 13, the interaction between two apolar bodies will therefore amount to about $52 \mathrm{~mJ} / \mathrm{m}^{2}$. This interaction is the so-called hydrophobic interaction. On the basis of the very crude estimate mentioned above, this is expected to be on the order of $0.13 k_{B} T$ per $\AA^{2}$.

A practical way of applying this framework to biomolecule interactions is based on the concept of accessible surface area (Lee 1971), which is defined as the area of the surface spanned by the center of a solvent molecule remaining in contact with the surface of a given molecule. A water molecule is usually modeled as a sphere of $1.4 \AA$ radius (Figure 6 ) . On the basis of this definition and of known free energies of transfer of aminoacids from an organic solvent to water, Chothia estimated at about $0.04 k_{B} T$ per $\AA^{2}$ the free energy required to expose hydrophobic residues on protein surfaces (Chothia 1974).

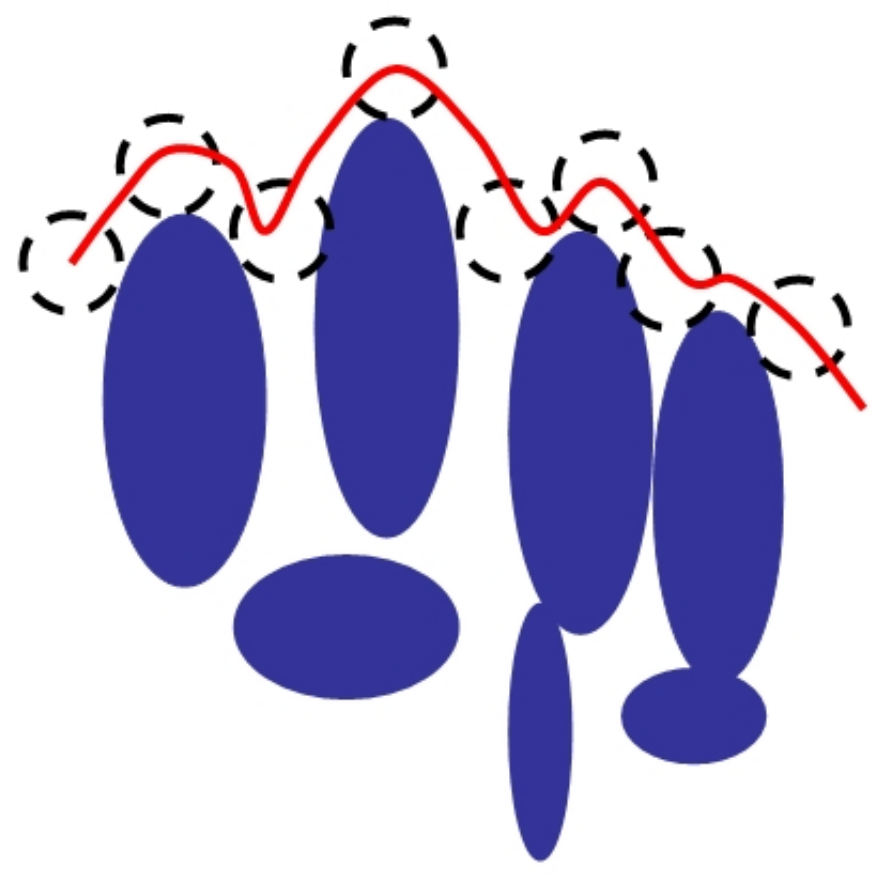

Figure 6: Accessible area. The accessible surface (red line) may be defined as the surface spanned by the center of a sphere representing a water molecule (broken contour) moving in contact with atoms constituting the protein and modeled as hard spheres with a known van der Walls radius (dark blue areas)

Other authors looked for more detailed estimates of the transfer free energy of individual chemical groups from an apolar to an aqueous environment in order to obtain a more accurate estimate of the energy of protein folding. The basic equations was : 


$$
\Delta F=\sum_{i} g_{i} A_{i}
$$

Where the summation extends over all groups i of the solute and $A_{i}$ is the conformationdependent accessible surface area. The hydration free energy of chemical groups such as $\mathrm{CH}_{3}, \mathrm{OH}$ or $\mathrm{C}=\mathrm{O}$ was estimated at about $0.03,0.29$ and $0.72 k_{B} T / \AA^{2}$ respectively (Ooi 1987).

In conclusion, in this very brief review, we summarized some basic concepts that are used as a crude framework to describe intermolecular forces. This will be the starting point for a discussion of the basic mechanisms of ligand-receptor association.

\subsection{General properties of ligand-receptor association}

While it would certainly be quite naive to look for universal properties of biomolecule interactions, it is useful to describe selected examples in order to help the reader get a reasonably realistic feeling for involved mechanisms. As indicated above, we shall essentially consider proteins.

\subsubsection{A static view of ligand-receptor complexes}

The contact area between two interacting proteins may be defined as the region that was accessible in isolated molecules and that is no longer accessible in complexes (assuming that the interaction did not trigger major morphological changes, which is usually the case). In many cases involving membrane receptors or antibodies, molecular association may result in a loss of accessible area comprised between about 800 and 1,600 $\AA^{2}$ (Chothia 1975, Jones 1996, Conte 1999). The interaction may involve between 10 and 30 side chains from each protein (Clackson 1995). Several questions are of interest:

Do all residues located in contact areas contribute a similar part to binding affinity? The answer is probably negative and extensive replacement of individual residues was used to try and assess the relative importance of each interaction. In a pioneering study made on the interaction between human growth hormone and its receptor (Cunningham 1989, Clackson 1995), it was concluded that more than $75 \%$ of the total binding energy was accounted by a central hydrophobic region essentially involving two trp residues: the replacement of each of these trp reduced the binding free energy by more than $7 k_{B} T$ (Clackson 1995). It was thus concluded that a few hot spots contributed most of the interaction energy. More recently, when a recombinant $\mathrm{T}$ cell receptor was made to interact with a series of nonapeptides bound by a same major histocompatibility complex molecule, the replacement of a single leucine with a valine was sufficient to increase the binding free energy by $2 k_{B} T$ and provoke a threefold decrease of the dissociation rate, and this difference resulted in nearly tenfold change of $\mathrm{T}$ lymphocyte activation potency (Aleksic 2010). In a patient with a bleeding disease, a replacement of a lysine with a valine resulted in 2.2 fold decrease of the dissociation rate between von Willebrand factor and GPIb $\alpha$ ligand under flow (Kumar 2003). In another study, the replacement of an alanine with a valine in a $\mathrm{T}$ cell receptor chain resulted in a binding free energy change of about $3 k_{B} T$ (Yang 2003). 
The authors emphasized that the affinity changes displayed cooperative rather than additive behaviour when they compared the effect of separate or concomitant changes of aminoacids at several positions (Yang 2003). An important point is that is some cases binding properties were changed following an alteration of an aminoacid residue located out of the contact area as identified on the basis of crystallographic studies.

Is a particular type of interaction favored in biologically relevant ligandreceptor couples ? As recently emphasized (Brock 2007), the role of electrostatics on binding affinities remains controversial, but a study of nearly 300 protein complexes led the authors to conclude that in most cases electrostatic forces did not strongly contribute to binding affinity. Indeed, there is a balance between desolvation energies required for molecular contact and attraction energies if there is a match between charges born by opposite surfaces. There are however some examples where electrostatic charges contribute significantly to binding efficiency. Electrostatic interactions contributed by 4 glutamic acid residues was estimated to account for $32 \%$ of the binding energy in the thrombin-hirudin couple (Stone 1989). Also, electrostatic interactions might be useful to give a proper orientation to approaching ligands and receptors, a phenomenon denominated as electrostatic steering (Kozack 1995). More recently, alanine scanning was used to study the influence of individual aminoacids on the binding to a talin oligopeptide of a 25-mer sequence of the membrane-proximal tail of several integrins. A general finding was that alanine replacement of acidic groups increased binding efficiency, while alanine replacement of basic groups slightly reduced this interaction (Gingras 2009), thus supporting the influence of charges in some cases of molecular interactions.

Further, there is much evidence supporting the view that hydrophobic bonds are responsable for an important part of binding affinities. (Chothia 1975, DeLano 2000, Conte 1999). In a compilation of 75 ligand-receptor complexes of known atomic structure, the fraction of apolar atoms was about $56 \%$. Hydrophobic bonds might thus contribute on the order of several tens of $k_{B} T$ to the binding free energy, on the basis of aforementioned estimates of contact areas and solvation free energies.

The average surface density of hydrogen bonds was estimated as one per $170 \AA^{2}$ in the series of 75 complexes mentioned above (Conte 1999). A recent study of the effect of a number of mutations on the interaction between a triacylated lipopeptide and a pattern recognition receptor led the authors to emphasize the importance of a network of hydrogen bonds in binding (Kajava 2010).

In conclusion, despite a number of studies based on a growing number of 3D structures, recognition interfaces between ligands and receptors did not display universal specific features, and it seems accepted that binding is a variable blend of polar and apolar interactions that will result in sufficient binding energy provided there is a proper match between the shape and charge distribution of interacting surfaces.

\subsubsection{Dynamics of ligand-receptor interaction}

A static view of the structure of biomolecule interfaces is not sufficient to provide a mechanistic understanding of molecular association. During the last decades, many investigators attempted to chose between two general concepts. 
According to the lock and key hypothesis, proteins behave as rigid bodies and recognition events require that two regions of the surfaces of interacting molecules be complementary enough to allow the formation of a closely packed interface. The main consequences of this hypothesis would be as follows : i) The 3D structures of free and bound receptors should be similar. This prediction can be tested more and more readily since structural databases contain a growing number of complex structures involving molecules that have already been studied in isolation. ii) Thus, the major requirement for bond formation would be that biomolecules encounter each other with a convenient orientation. The loss of translational entropy associated to bond formation would be rather limited, and might be compensated for by the entropy increase associated to the formation of hydrophobic bonds.

The induced fit hypothesis suggests that biomolecule association should involve an adaptation of at least one of interacting molecules in order to match the structure of the opposing surface. In this case, bond formation would be expected to involve a number of intermediate states.

The conformation selection hypothesis is only quantitatively different from the previous one : it is suggested that any protein will display a number of different conformations. Association between a receptor and a ligand would require that molecular encounter occurred between complementary conformations. In this case, a high proportion of encounters could not be conducive to association, and the rate of bond formation would be sigificantly lower.

It must be emphasized that the difference between the induced fit and conformation selection are only quantitative: in the latter case, the energies of different conformations are sufficiently similar to make each conformation detectable in absence of association. In the former case, the free energy of the bound conformation is too high to be detectable in absence of stabilization. During the last decades, a number of investigations disclosed the complexity of protein behaviour: while crystallographic studies conveyed the feeling that many proteins displayed a fairly rigid conformation, with a precise localization of atoms at the $\AA$ scale, dynamic studies showed that proteins were in fact dynamic objects with an enormous number of states and substates (Ansari 1985). Thus, the discrimination between aforementioned views may seem somewhat naive. However, this is often a basis for interpreting many experimental data. This point will now be clarified by some examples.

$\mathrm{T}$ cell receptor (TCR) association with pMHC ligand has already been mentioned in the first section of this review as an important model for biomolecule recognition and it was felt that structural studies should illuminate the recognition process. Early studies suggested that the TCR exhibited extremely poor shape complementarity with the peptide, as exemplified by the interaction between $2 \mathrm{C}$ TCR and $H-2 K^{b}-d E V 8$ ligand (Garcia 1998). In a later thermodynamic study, it was indeed suggested that the induced fit of TCR to a peptide could allow the required discriminatory power (Boniface 1999). A few years later, a structural study made on another TCR (2B4) led the investigators to the conclusion that the initial TCR/pMHC interaction essentially involved an interaction between the TCR and MHC, and that a later phase involved an interaction with the TCR and the peptide bound to the MHC (Wu 2002). The authors emphasized that different MHCs could display different behaviour. However, a 
few years later, the authors of a review based on 24 TCR/pMHC complexes acknowledged that crystal structures did not allow to define a common mechanism for the interaction and ongoing signaling phenomena (Rudolph 2006). Finally, kinetic studies of pMHC-TCR association with surface plasmon resonance led to the conclusion that binding was a multiphasic reaction, suggesting either induced fit or conformation selection (Gakamsky 2004). The need for TCR to recognize a variety of peptide might require a particular role for molecule flexibility. However, there is also experimental evidence for the importance of molecular flexibility in less variable models of biomolecular recognition. Thus, the interaction between $\operatorname{IgE}$, the antibodies with a major role in allergic reactions, and IgE receptors was reported to involve alterations of receptor structure, as revealed with NMR spectra and release of bound fluorescent probes (Price 2005). Other NMR-based studies yielded similar conclusions : calmodulin is a cytoplasmic molecule that plays a major role in mediating functional consequences of intracellular calcium changes by interacting with a number of targets. It was reported that i) ligand-binding resulted in marked changes of calmodulin conformational dynamices, and ii) apparent changes of conformational entropy were linearly related to the change of overall binding entropy, showing that binding-associated changes of protein conformation significantly contributed to binding free energy (Frederick 2007). In another study made on the interaction between cytokine interleukin-18 (IL-18) and specific antibodies, crystallographic studies revealed important displacements, up to $10 \AA$, of some specific residues (Argiriadi 2009) Another direct proof of the importance of receptor flexibility was obtained with the surface forces apparatus, a device allowing to quantify the distance between decorated mica surfaces with $1 \AA$ resolution. This was used to study the interaction between DC-SIGN, a pattern recognition receptor, and oligosaccharide ligands. When surfaces bearing receptors and ligands were approached and retracted, a $2.4 \mathrm{~nm}$ alteration of receptor length was observed (Menon 2009). Note that in this case geometric changes might involve binding sites or protein domains linking them to the surface.

In contrast to the $\mathrm{TCR} / \mathrm{pMHC}$ interaction, the recognition of a number of ligands by immunoreceptor NKG2D was claimed to involve only rigid adaptation, as suggested by higher binding rate and a more favorable binding entropy (McFarland 2003). This supports the previous report that association involved only limited changes of conformation in 75 complexes studied with crystallography (Conte 1999).

In conclusion, there is a growing evidence that proteins are highly flexible objects, and increasingly sensitive analytical methods are expected to reveal more and more structural or dynamical changes associated to bond formation. The relative role of these changes in binding free energy may however display wide variations when different biological systems are studied.

\subsubsection{Mechanisms influencing the specificity of biomolecule interaction}

As indicated above, specificity is an essential property of biomolecule association. The information described in the preceding section will be a basis to describe more precisely the meaning of molecular specificity. Indeed, there are several potential mechanisms that might account for the capacity of a given receptor to bind different ligands. We 
shall list them, then some informative examples will be given to support the significance of these mechanisms.

Possible mechanisms for cross reactivity i) Different molecules may share a common binding region. This may be a consequence of a common origin, since it is known that proteins share a limited number of basic folds. Alternatively, this may be a consequence of some kind of convergent evolution (DeLano 2000). Finally, cross reactivity may be due to a random similarity of unrelated molecules: as an example, Kabat reported a similarity of three- dimensional models of purin-6-oylglycine and 2'deoxyadenylic acid, which might provide an explanation for observed cross reactions between antisera to purin-6-oyl-bovine serum albumin and DNA (Kabat 1966). ii) In a given contact region between a receptor and different ligands, different hot-spots might account for the binding of different molecules. iii) A biomolecule might display sufficient flexibility to bind to different molecules. iv) A given receptor might be endowed with a number of unrelated binding sites specific for different molecules. So-called adapters are but an example.

Examples of promiscuous receptors A crystallographic study revealed that a monoclonal antibody (CB4-1) could bind 5 unrelated peptides on the same binding region (paratope), but these peptides formed different contacts (Kramer 1997).

Other authors reported on a single antibody that could bind several unrelated antigens. They performed both X-ray crystallography and kinetic studies, and provided strong proof of the occurrence of conformational diversity and induced fit (James 2003). The hypothesis that antibody promiscuity might be due to high flexibility is also reported by a comparison between two mannopyranoside antibodies, $1 \mathrm{H} 7$ and 2D10. The antibody with a narrow specificity, 1H7, displayed much lower flexibility than promiscuous antibody 2D10 (Krishnan 2008).

In an other model, the use of spectroscopic methods such as circular dichroism and fluorescence measurements revealed that hyaluronan-binding protein 1, an oligomeric protein with a capacity to bind different ligands such as $\mathrm{C} 1 \mathrm{q}$ and hyaluronan, displayed marked structural changes when the ionic strengh was altered concomitantly with a change of ligand specificity (Jha 2003). The importance of conformal diversity was also advocated in a study made on peptide recognition by a chicken major histocompatibility complex molecule (Koch 2007).

The involvement of shared structures in some cases of recognition multiplicity is supported by a study of the $\alpha X \beta 2$ integrin, a leukocyte receptor with a capacity to detect danger signals : recognition seemed to involve carboxylic groups that seemed to appear more frequently on damaged proteins (VorupJensen 2005). Potential mechanisms of receptor promiscuity are displayed on figure 7

Conclusion While specificity is a hallmark of biomolecule interactions, promiscuity is also a common finding, as testified by the multiplicity of interactions found in databases.

In addition, the data presented in this brief review illustrate the diversity of situations and the complexity of the binding mechanisms that involve multiple confor- 




Figure 7: Possible mechanisms of cross reactivity. Several different mechanisms were shown to result in receptor promiscuity. (A) Different ligands may form different elementary bonds in a same binding site (Kramer 1997). (B) A molecule may display several unrelated binding sites on its surface. (C) A binding site may be flexible and accomodate different ligands (James 2003). bf (D) Two unrelated molecules may display some local similarity (Kabat 1966).

mational changes and may depend on structural details of molecular surfaces at the nanometer scale. Therefore, there is clearly a need for a more accurate description of protein behaviour to interpret more and more refined experimental data. A tentative way of approaching this goal consists of using computer simulation to try and correlate subtile behavioural patterns to structural details. This point will be considered in the following section.

\subsection{Information yielded by computer simulation}

A conclusion of the results described in previous sections is that i) experimental dissection of bond formation and dissociation at the single molecule level brought an accurate description of many models of biomolecule recognition as highly multiphasic processes with complex force dependence. ii) Experimental study of isolated protein molecules or molecular complexes with techniques such as X-ray crystallography or NMR yielded a wealth of structural data with nearly $\AA$ accuracy. iii) Currently available knowledge of intermolecular forces yielded a relatively intuitive understanding of the relationship between protein structure and binding behaviour. However, this remains too approximative to allow accurate prediction of outcomes in well defined situations. iv) Current theoretical work aims at finding more detailed links between structure and function. However, it is well known that ... In the real world, exact solutions are the notable exception (Rapaport 2004). Further, analytic solutions may be too complicated to be of real use. 
During the past decades, the increasing availability and power of computers triggered the development of simulation as a general tool for trying to bring insights into models that are too complex to allow simple mathematical description. The basic idea consists of observing the behaviour of a model system containing enough ingredients to mimic a realistic situation. We shall now give a few examples to illustrate the kind of information that can be obtained with this approach. However, a word of caution as found in the introduction of a recent treatise of molecular dynamics simulation may be useful: ... Simulations must be kept honest, because seing is believing, and animated displays can be very convincing irrespective of their veracity (Rapaport 2004). We shall sequentially consider two approaches of growing complexity.

Simulations as numerical solutions of simple equations. Even fairly simple models cannot be described with plain mathematical formula. As an example, when flow chambers are used to study the motion of receptor-coated microspheres near ligand bearing surfaces, a quantitative determination of the rate of bond formation requires a knowledge of the frequency and duration of molecular encounters. The distance between spheres and surfaces must therefore be known with millisecond and nanometer accuracy, which is difficult to achieve with present day techniques. This was an incentive to mimic the motion of microspheres near surfaces in presence of hydrodynamic shear and account for brownian motion and hydrodynamic interactions between the sphere and the wall (Pierres 1998, Pierres 2001). This approach was necessary to obtain quantitative information on the kinetic rate of bond formation (Pierres 1998). The validity of simulations was subjected to theoretical (Pierres 2001) and experimental (Robert 2008) check. In a later study, numerical simulations were found useful to demonstrate that the bond formation between a ligand and a receptor might be view as the progression of the complex along a linear segment of a rugged energy landscape rather than the passage across an energy barrier, as was usually considered (Robert 2009).

Simulating complex systems: molecular dynamics and related approaches The basic idea of molecular dynamics was reported by Alder and Wainwright (Alder 1957) in the late fifties. They modeled a system made of hard spheres in a box, starting with a random configuration defined as positions and velocities. Then they applied the law of mechanics to follow the displacements of all spheres through a series of ultrashort steps, and the new positions and velocities of spheres were recorded at each step. Fourteen years later, the motion of several tens of model water molecules interacting with more realistic potentials was similarly simulated (Rahman 1971). The continual growth of computer power allowed investigators to study models of increasing complexity, until they were able to simulate interactions between proteins molecules including tens of thousands of atoms, and surrounded by hundreds of water molecules. The most detailed approach consisted of modeling interactions between pairs of atoms or chemical groups with semi-empirical potential functions derived from quantum chemical considerations as well as experimental data. Using femtoseconds time steps, this method currently allows the observation of objects as complex as proteins during less than a microsecond, which is still insufficient to account for molecular interactions. 
Calculations are performed with arrays of computers, using a few standard software packages such as CHARMM (Brooks 2009), Gromos (Christen 2005), Rosetta (Das 2008) or the freely available Xplor-NIH package (Schwieters 2003). In the following sections, we shall first discuss rapidly some limitations of the method and tricks suggested to progress. Then we shall describe a few selected examples to illustrate the kind of information that can be obtained. More information can be found in aforementioned papers and standard treatises (Rapaport 2004).

\subsubsection{Limitations and technical advances.}

As shown above, there is a need to extend the range of accessible domains to take real advantage of computer simulations. Here are some noticeable points.

long distance cut-off of interactions Since most interactomic forces exhibit a rapid decay with distance, it seemed reasonable to neglect interactions at distance higher than some arbitrary cut-off. A useful point is that artefacts may be avoided by using e.g. a smoothened cutoff or Ewald summation technique (Hansen 1973, Johnson 2007, Brooks09).

Poisson-Boltzmann statistics.. Due to the complexity of solvent effect, it was tempting to account for water dielectric constant by considering individual molecules. Unfortunately, this is highly time consuming. It was suggested to use numerical solution of Poisson-Boltzmann equation to calculate the potential on the surface of proteins (Honig 1995).

Protein flexibility. A major difficulty is the formidable number of configurations available for proteins. Initial docking software thus treated proteins as rigid objects (Shoichet 1993, Fahmy 2002), which greatly increased calculation speed, but is also an important limitation, as discussed above. This was an incentive to elaborate clever research algorithm to sample a sufficient variety of conformations within a reasonable amount of time. Examples are the use of so-called "genetic search algorithms" which allowed successful simulation of association of couples such as methotrexate and dihydrofolate reductase, or galactose and L-arabinose binding protein (Jones 1995).

Coarse-grained potential. A major limitation of molecular dynamics is that the passage across significant energy barriers may require an excessive amount of time. This difficulty may be overcome by smoothing barriers to allow a more rapid exploration of more extensive configurations. In a later step, a more refined potential may be used (Moritsugu 2007).

Brownian dynamics and hybrid simulation Another way to overcome the time limitation of molecular simulations consists of combining deterministic equations accounted for ultrashort step with possibly random equations accounting for a high number of steps. For example, the displacement of a water molecule during a femtosecond interval would be a few thousands of $\AA$ if the molecule velocity is on the order of a few hundreds of meter/s, corresponding to $\left(k_{B} T / m\right)^{1 / 2}$, where $m$ is the molecule mass. It may be more rapid to generate random diffusive displacements according to the laws of diffusion (Northrup 1992). In a later study, in order to simulate the dissociation of a streptavidin-biotin bond with an atomic force microscope, the cantilever motion was described with equations from classical mechanics, while the molecular deformations 
and motion were simulated with standard molecular dynamic approach. This approach was rightly denominated as hybrid simulation (Zhou 2006).

Reliability check. A major problem with any powerful method is that it is difficult to check its reliability when it yields data that cannot be obtained with any alternative approach. This difficulty is of major importance with computer simulation due to the number of required assumptions. A marked progress consisted of organizing systematic checks of predictions by asking investigators to study selected model systems the structure of which was currently studied. It was thus possible to compare the predictions yielded by different softwares to the experimental data that were available soon thereafter. This approach is the basis of CASP (critical assessment of structure prediction) and CAPRI (critical assessment of predicted interactions) (SchuelerFurman 2005, FernandezRecio 2010).

\subsubsection{New information on molecular association may be provided by com- puter simulations}

Simulation of force-driven bond rupture. Soon after the report of experimental rupture of individual avidin-biotin bonds with an atomic force microscope (Florin 1994, Lee 1994), computer simulations were performed to simulate unbinding over periods of up to 500 ps (Grubmuller 1996, Izrailev 1997). Interestingly, when the biomembrane force probe allowed a tentative dissection of the unbinding path (Merkel 1999), the authors could compare results from simulation (Izrailev 1997) to the tentative energy landscape they were able to build on the basis of their experimental data (see Fig. 4 of (Merkel 1999)). As another example, K. Shulten and D. Leckband investigated the force-induced rupture of the CD2-CD58 complex (Bayas 2003). This association might play a role in the interaction between $\mathrm{T}$ lymphocytes and antigen presenting cells. The authors found that two different dissociation mechanisms might occur depending on the loading rate. At a high loading rate of 35 or $70 \mathrm{pN} / \mathrm{ps}$, the proteins began unfolding before being separated. As a tenfold lower loading rate, the proteins separated before unfolding. Further, the authors concluded that salt bridges were the primary determinants of tensile strength.

A few years later, computer simulations (Lou 2006, Gunnerson 2009) were used to investigate the behaviour of so-called catch bonds. These bonds were predicted to display a paradoxical force sensitivity with lifetime increase in presence of a disruptive force (Dembo 1988), and they were evidenced with flow chambers (Thomas 2002, Marshall 2003) and atomic force microscopy (Marshall 2003). Soon thereafter, a theoretical report showed that this behaviour was consistent with a two-pathway model of bond rupture (Pereverzev 2005). The following year, a report based on crystallographic studies and computer simulation suggested a relationship between L-selectin behaviour and the existence of two conformations, with a possible effect of forces on transition between these two states (Lou 2006). In a later paper (Lou 2007), the authors suggested a more precise mechanism.

Dissecting the kinetics of molecular interactions.. The interaction between acetylcholinesterase and tetramethylammonium (TMA), a molecule resembling the bulkiest part of acetylcholine, was studied with molecular dynamics. The authors 
concluded that local conformational fluctuations were required to allow the ligand passage (Bui 2003). More recently, molecular dynamics was used to investigate the mechanisms of interactions between tyrosine kinases and a monoclonal antibody (Aleksandrov 2010): the authors concluded that binding preferences were determined by conformational selection.

Predicting molecular interactions A major goal of molecular simulation is to predict molecular interactions. As already mentioned, molecular simulations have been used for years to try and predict molecular associations (Jones 1995, Das 2009) in addition to other knowledge-based approaches (Chuang 2008, Keiser 2009). As indicated above, it would be most useful to be able to predict molecular interactions and the present state of the art is reviewed is CAPRI rounds (FernandezRecio 2010). Successes go on being reported in present day literature (Das 2009, Goley 2010).

\section{Conclusion}

Biomolecule recognition is a process of outstanding biological importance that has been studied for decades with standard physical chemical methods and concepts, based on the determination of affinity constants and kinetic association and dissociation rates. Results were interpreted within the framework of thermodynamics, statistical physics and physical chemistry as elaborated nearly a century ago. More recently, the experimental dissection of molecular interactions at the single bond level, increasing availability of structural data with nearly $\AA$ resolution, and development of simulation methods with a power matching protein complexity, made it conceivable to describe molecular interactions with much improved accuracy, with a deeper understanding of the force sensitivity and transition between a multitude of substates. In parallel, the progress of our understanding of all the complexity of biological systems is an incentive to use this new kind of knowledge to achieve a better understanding of cell function. It is hoped that the development of dynamic force spectroscopy will be a substantial factor of progress along this line.

\section{References}

Alberts, B., A. Johnson, J. Lewis, M. Raff M, K. Roberts, and P. Walter. 2008. Molecular Biology of the Cell 5th edition, New York:Garland.

Aleksic M., O. Dushek, H. Zhang, E. Shenderov, J-L. Chen, V. Cerundolo, D. Coombs, and P. A. van der Merwe. 2010. Dependence of T cell antigen recognition on T cell receptorpeptide MHC confinement time. Immunity 32:163-174.

Alon, R., D. A. Hammer, and T. A. Springer. 1995). Lifetime of P-selectin-carbohydrate bond and its response to tensile force in hydrodynamic flow. Nature 374 : 539-542. (Correction: Nature 377:86, 1995).

Ansari, A., J. Berendzen, S. F. Bowne, H. Frauenfelder, I. E. T. Iben, T. B. Sauke, E. Shyamsunder, and R. T. Young. 1985. Protein states and proteinquakes. Proc. Natl. Acad. Sci. USA 82:5000-5004.

Argiriadi, M. A., T. Xiang, C. Wu, T. Ghayur, and D. W. Borhani. 2009. Unusual water-mediated antigenic recognition of the proinflammatory cytokine Interleukin-18. $J$. 
Biol. Chem. 284:24478-24489.

Astrof, N. S., A. Salas, M. Shimaoka, J. F. Chen, and T. A. Springer. 2006. Importance of force linkage in mechanochemistry of adhesion receptors. Biochemistry 45:15020-15028.

Badis, G., M. F. Berger, A. Anthony, A. A. Philippakis, S. Talukder, A. R. Gehrke, S. A. Jaeger, E. T. Chan, G. Metzler, A. Vedenko, X. Chen, H. Kuznetsov, C-F. Wang, D. Coburn, D. E. Newburger, Q. Morris, T. R. Hughes and M. L. Bulyk. 2009. Diversity and Complexity in DNA Recognition by Transcription Factors. Science 324: 1720-1723.

Baumgartner, W., P. Hinterdorfer, W. Ness, A. Raab A, D. Vestweber, H. Schindler, and D. Drenckhahn. 2000. Cadherin interaction probed by atomic force microscopy. Proc. Natl. Acad. Sci. USA 97 : 4005-4010.

Bayas, M. V., K. Schulten, and D. Leckband. 2003. Forced detachment of the CD2-CD58 complex. Biophys. J. 84:2223-2233.

Bell, G. I. 1978. Models for the specific adhesion of cells to cells. Science 200:618-627.

Bell, G. I., M. Dembo, and P. Bongrand. 1984. Cell adhesion : competition between nonspecific repulsion and specific bonding. Biophys. J. 45:1051-1064.

Best, R. B., and G. Hummer. 2005. Reaction coordinates and rates from transition paths. Proc. Natl. Acad. Sci. USA 102:6732-6737.

Blow, N. 2009. Untangling the protein web. Nature. 460:415-418.

Bongrand, P. and G. I. Bell. 1984. Cell-Cell Adhesion: parameters and possible mechanism. In Cell Surface Dynamics. Concepts and Models. eds A. S. Perelson, C. DeLisi, and F. W. Wiegel. 459-493. New York: Marcel Dekker.

Bongrand, P., P. M. Claesson, and A. S. G. Curtis (eds). 1994 Studying cell adhesion. Berlin:Springer-Verlag.

Bongrand, P. and B. Malissen. 1998. Quantitative aspects of T-cell recognition : from within the antigen-presenting cell to within the T cell. Bioessays 20:412-422.

Bongrand, P. 1999. Ligand-receptor Interactions. Rep. Prog. Phys. 62:921-968.

Boniface, J. J., Z. Reich, D. S. Lyon, and M. M. Davies. 1999. Thermodynamics of T cell receptor binding to peptide-MHC : evidence for a general mechanism of molecular scanning. Proc. Natl. Acad. Sci. USA 96:1446-11451.

Brock, K., K. Talley, K. Coley, P. Kundrotas, and E. Alexov. 2007. Optimization of electrostatic interactions in protein-protein complexes. Biophys. J. 93:3340-3352.

Brooks, B R., C. L. Brooks III, A. D. MacKerell Jr., L. Nilsson, R. J. Petrella, B. Roux, Y. Won, G. Archontis, C. Bartels, S. Boresch, A. Caflisch, L. Caves, Q. Cui, A. R. Dinnr, M. Feig, S. Fishcher, J. Gao, M. Hodoscek, W. Im, K. Kuczera, T. Lazaridis, J. Ma, V. Ovchinnikiv, E. Paci, R. W. Pastor, C. B. Post, J. Z. Pu, M. Schaefer, B. Tidor, R. M. Venable, H. L. Woodcock, X. Wu, W. Yang, D. M. York, and M. Karplus. 2009. J. Comput Chem. 30:1545-1614.

Brunk, D. K., D. J. Goetz and D. A. Hammer. 1996. Sialyl Lewisx/E-selectin-mediated rolling in a cell free system. Biophys. J. 71:2902-2907.

Bunch, T. A. 2010. Integrin alphaIIb beta3 activation in chinese hamster ovary cells and platelets increases clustering rather than affinity. J. Biol. Chem. 285:1841-1849.

Capo, C., P. Bongrand, A. M. Benoliel, and R. Depieds. 1978. Dependence of phagocytosis on strength of phagocyte-particle interaction. Immunology 35:177-182.

Chan, P. Y., M. B. Lawrence, M. L. Dustin, L. M. Ferguson, D. E. Golan, and T. A. Springer. 1991. Influence of receptor lateral mobility on adhesion strengthening between membranes containing LFA-3 and CD2. J. Cell Biol. 115 : 245-255.

Chaurasia, G., S. Malhotra, J. Russ, S. Schnoegl, C. Hnig C, E. E. Wanker, and M. E. Futschik. 2008. UniHI 4 : new tools for query, analysis and visualization of the human 
protein-protein interactome. Nucleic acids research 37 : 657-660.

Chen C. S., M. Mrksich, S. Huang, G. M. Whitesides, and D. E. Ingber. 1997. Geometric control of cell life and death. Science 276:1425-1428.

Chen, S., and T. A. Springer. 2001. Selectin receptor-ligand bonds : formation limited by shear rate and dissociation governed by the Bell model. Proc. Natl. Acad. Sci. USA. 98:950-955.

Chen, W., E. A. Evans, R. P. McEver, and C. Zhu. 2008. Monitoring receptor-ligand interactions between surfaces by thermal fluctuations. Biophys. J. 94:694-701.

Chen W, V. I. Zarnitzyna, K. K. Sarangapani, J. Huang, and C. Zhu. 2008a. Measuring receptor-ligand binding kinetics on cell surfaces: from adhesion frequency to thermal fluctuation methods. Cell. Mol. Bioeng. 1:276-288.

Chesla, S. E., P. Selvaraj, and C. Zhu. 1998. Measuring two-dimensional receptor-ligand binding kinetics by micropipette. Biophys. J. 75:1553-1572.

Chilkoti, A., T. Boland, B. D. Ratner, and P. S. Stayton. 1995. The relationship between ligand binding thermodynamics and protein-ligand interaction forces measured by atomic force microscopy. Biophys. J. 69:2125-2130.

Choi, H., H. Kang, and H. Park. 2010. New angle-dependent potential energy function for backbone-backbone hydrogen bond in protein-protein interactions. J. Comput. Chem. 31:897-903.

Chothia, C. 1974. Hydrophobic bonding and accessible surface area in proteins. Nature 248:338-339.

Chothia, C., and J. Janin. 1975. Principles of protein-protein recognition. Nature 256:705-708.

Christen, M., P. H. Hnenberger, D. Bakowies, R. Baron, R. Brgi, D. P. Geerke, T. N. Heinz, M. A. Kastenholz, V. Krutler, C. Oostenbrink, C. Peter, D. Trzesniak. 2005. The GROMOS software for biomolecular simulation: GROMOS05. J. Comput. Chem. 26:17191751 .

Chuang, G-Y., D. Kozakov, R. Brenke, S. R. Comeau, and S. Vajda. 2008. DARS (Decoys As the Reference State) potentials for protein-protein docking. Biophys. J. 95:4217-4227.

Clackson T., and J. A. Wells. 1995). A hotspot of binding energy in a hormone-receptor interface. Science $267: 383-386$.

Connelly, P. R., R. A. Aldape, F. J. Bruzzese, S. P. Chambers, M. J. Fitzgibbon, M. A. Fleming, S. Itoh, D. J. Livingston, M. A. Navia, J. A. Thomson, and K. P. Wilson. 1994. Enthalpy of hydrogen bond formation in a protein-ligand binding reaction. Proc. Natl. Acad. Sci. USA 91:1964-1968.

Conte, L. L., C. Chothia, and J. Janin. 1999. The atomic structure of protein-protein recognition sites. J. Mol. Biol. 285:2177-2198.

Creighton, T. E. Proteins - Structure and Molecular Properties, 1st edn. (Freeman, New York, 1983).

Cretel, E., D. Touchard, A. M. Benoliel, P. Bongrand, and A. Pierres. 2010. J. Phys. Condensed matter 22:194107.

Cunningham, B. C., and J. A. Wells. 1989. High-resolution epitope mapping of hGHReceptor interactions by alanine scanning mutagenesis. Science 244:1081-1085.

Das, R., and D. Baker. 2008. Macromolecular modeling with Rosetta. Ann. Rev. Biochem. 77:363-382.

Das, R., I. Andr, Y. Shen, Y. Wu, A. Lemak, S. Bansal, C. H. Arrowsmith, T. Szyperski, and D. Baker. 2009. Simultaneous prediction of protein folding and docking at high resolution. Proc. Natl. Acad. Sci. USA. 106:18978-18983. 
DeLano, W. L., M. H. Ultsch, A. M. de Vos, and J. A. Wells. 2000. Convergent solutions to binding at a protein-protein interface. Science 287:1279-1283.

Del Rio, A., R. Perez-Jimenez, R. Liu, P. Roca-Cusachs, J. M. Fernandez, and M. P. Sheets. 2009. Stretching single talin rod molecules activates vinculing binding. Science 323:638-641.

Dembo, M., D. C. Torney, K. Saxman, and D. Hammer. 1988. The reaction-limited kinetics of membrane-to-surface adhesion and detachment. Proc. Roy. Soc. Lond. B 234:5583.

Diz, R., S. K. McCray, and S. H. Clarke. 2008. B cell receptor affinity and B cell subset identity integrage to define the effectiveness, affinity threshold, and mechanism of anergy. $J$. Immunol. 181:3834-3840.

Dobereiner, H. G., B J. Dubin-Thaler, J. M. Hofman, H. S. Xenias, T. N. Sims, G. Giannone, M. Dustin, C. H. Wiggins, and M. P. Sheetz. 2006. Lateral membrane waves constitute a universal dynamic pattern of motile cells. Phys. Rev. Let. 97:038102.

Dudko, O. K., G. Hummer, and A. Szabo. 2006. Intrinsic rates and activation free energies from single-molecule pulling experiments. Phys. Rev. Letters 96:108101.

Dukdo OK, G Hummer, A Szabo. 2008. Theory, analysis and interpretation of singlemolecule force spectroscopy experiments. Proc. Natl. Acad. Sci. USA 105:15755-15760.

Dustin, M. L., S. K. Bromley, M. M. Davis, and C. Zhu. 2001. Identification of self through two-dimensional chemistry and synapses. Ann. Rev. Cell Dev. Biol. 17 : 133-157.

Eisenberg, D, and W. Kauzmann. 1969. The structure and properties of water. Oxford: Oxford University Press.

Engler, A. J., S. Sen, H. L. Sweeney, and D. E. Discher. 2006. Matric elasticity directs stem cell lineage specification. Cell 126:677-689.

Evans, E., D. Berk, and A. Leung. 1991. Detachment of agglutinin-bonded red blood cells. I - Forces to rupture molecular-point attachments. Biophys. J. 59:838-848, 1991.

Evans, E., R. Merkel, K. Ritchie, S. Tha, A. Zilker. 1994. Picoforce method to probe submicroscopic actions in biomembrane adhesion. In Studying Cell Adhesion, eds P. Bongrand, P. M. Claesson, and A. S. G. Curtis. 125-139. Berlin: Springer-Verlag.

Evans, E., and K. Ritchie. 1997. Dynamic strength of molecular adhesion bonds. Biophys. J. $72: 1541-1555$.

Evans, E., and K. Ritchie. 1999. Strength of a weak bond connecting flexible polymer chains. Biophys. J. 76:2439-2447.

Evans, E. 2001. Probing the relation between force - lifetime - and chemistry in single molecular bonds. Annu. Rev. Biophys. Biomol. Struct. 30:105-128.

Evans E., A. Leung, D. Hammer, and S. Simon. 2001. Chemically distinct transition states govern rapid dissociation of single L-selectin bonds under force. Proc. Natl. Acad. Sci. USA. 98:3784-3789.

Eyring, H. 1935. The activated complex in chemical reactions. J. Chem. Phys. 3 : 107-115.

Eyring, H., J. Walter, and G. E. Kimball. 1944. Quantum Chemistry New York: Wiley.

Fahmy, A., and G. Wagner. 2002. TreeDock: a tool for protein docking based on minimizing van der Waals energies. J. Am. chem. Soc. 124:1241-1250.

Faix, J., and K. Rottner. 2006. The making of filopodia. Current Opinion Cell Biol. 18:18-25.

Fernandez-Recio, J., and M. J. E. Sternberg. 2010. The 4th meeting on the Critical Assessment of PRedicted Interaction (CAPRI) held a the Mare Nostrum, Barcelona. Proteins. 78:3065-3066. 
Florin, E. L., V. T. Moy, and H. E. Gaub. 1994. Adhesion forces between individual ligand-receptor pairs. Science $264: 415-417$.

Folkman, J., and A. Moscona .1978. Role of cell shape in growth control.

Nature 273:345-349.

Foote, J. and C. Milstein. 1991. Kinetic maturation of an immune response. Nature 352:530-532.

Frederick, K. K., M. S. Marlow, K. G. Valentine, and A. J. Wand. 2007. Conformational entropy in molecular recognition by proteins. Nature 448:325-330.

Freund, L. B. 2009. characterizing the resistance generated by a molecular bond as it is forcibly separated. Proc. Natl. Acad. Sci. USA 106:8818-8823.

Gakamsky, D. M., I. F. Luescher, and I. Pecht. 2004. T cell receptor-ligand interactions: a conformational preequilibrium or an induced fit. Proc. Natl. Acad. Sci. USA 101:9063-9066.

Garcia, K. C., M. Degano, L. R. Pease, M. Huang, P. A. Peterson, L. Teyton, and I. A. Wilson. 1998. Structural basis of plasticity in T cell receptor recognition of a self peptideMHC antigen. Science 279:1166-1172.

Gingras, A. R., W. H. Ziegler, A. A. Bobkov, M. G. Joyce, D. Fasci, M. Himmel, S. Rothemund, A. Ritter, J. G. Grossmann, B. Patel, N. Bate, B. T. Goult, J. Emsley, I. L. Barsukov, G. C. K. Roberts, R. C. Liddington, M. H. Ginsberg, and D. R. Critchley. 2009. Structural determinants of integrin binding to the talin rod. J. Biol. Chem. 284. 8866-8876.

Glinsky, VV, G. V. Glinsky, O. V. Glinskii, V. H. Huxley, R. Turk JR, V. V. Mossine, S. L. Deutscher, K. J. Pienta, and T. P. Quinn. 2003. Intravascular metastatic cancer cell homotypic aggregation at the sites of primary attachment to the endothelium. Cancer Res. 63:3805-3811.

Glynn, E., and M. V. Steward. eds. 1977. Immunochemistry: An advanced textbook 2nd edition. New york: Wiley. 240.

Grubmuller, H., B. Heymann, and P. Tavan. 1996. Ligand binding: molecular mechanics calculation of the streptavidin-biotin rupture force. Science 271:997-999.

Gunnerson, K. N., Y. V. Pereverzev, and O. V. Prezhdo. 2009. Atomistic simulation combined with analytic theory to study the response of the P-selectin/PSGL-1 complex to an external force. J. Phys. Chem. B 113:2090-2100.

Hanggi , P., P. Talkner, and M. Borkovec. 1990. Reaction-rate theory : fifty years after Kramers. Rev. Mod. Phys. 62: 251-341.

Hansen, J-P., 1973. Statistical mechanics of dense ionized matter. I. Equilibrium properties of the classical one-component plasma. Phys. Rev. A 8: 3096-3109.

Hinterdorfer, P., W. Baumgartner, H. J. Gruber, K. Schilcher, and H. Schindler. 1996. Detection and localization of individual antibody-antigen recognition events by atomic force microscopy. Proc. Natl. Acad. Sci. USA 93 : 3477-3481.

Homouz, D., M. Perham, A. Samiotakis, M. S. Cheung, and P. Wittung-Stafshede. 2008. Crowded, cell-like environement induces shape changes in aspherical protein. Proc. Natl. Acad. Sci. USA 105:11754-11759.

Honig, B., and A. Nicholls. 1995. Classical electrostatics in biology and chemistry. Science 268:1144-1149.

Huang, J., V. Zarnitsyna, B. Liu, L. J. Edwards, N. Jiang, B. D. Evavold BD, and C. Zhu. 2010.

The kinetics of two-dimensional TCR and pMHC interactions determine T-cell responsiveness. Nature 464:932-936.

Huppa, J. B., M. Axmann, M. A. Mortelmaier, B. F. Lillemeier, E. W. Newell, M. 
Brameshuber, L. O. Klein, G. J. Schutz, and M. M. Davis. 2010. TCR-peptide-MHC interactions in situ show accelerated kinetics and increased affinity. Nature 463: 963-970.

Israelachvili, J. N. 1991. Intermolecular and surface forces, 2nd Edition, New York: Academic Press.

Izrailev, S., S. Stepaniants, M. Balsera, Y. Oono, and K. Schulten. 1997. Molecular dynamics study of unbinding of the avidin-biotin complex. it Biophys. J. 72:1568-1581.

Jaalouk, D. E., and J. Lammerding. 2009. Mechanotransduction gone awry. Nature Rev. Mol. Cell Biol. 10:63-73.

James, L. C., P. Roversi, and D. S. Tawfik. 2003. Antibody multispecificity mediated by conformational diversity. Science 299:1362-1367.

Janin, J. 1996. Quantifying biological specificity: the statistical mechanics of molecular recognition. Proteins 25:438-445.

Jay, P. Y., P. A. Pham, S. A. Wong, and E. L. Elson. 1995. A mechanical function of myosin II in cell motility. J. Cell Sci. 108:387-393.

Jha, B. K., D. M. Salunke, and K. Datta. 2003. Structural flexibility of multifunctinal HABP1 may be important for regulating its binding to different ligands.J. Biol. Chem. 278:27464-27472.

Jones, G., P. Willett, and R. C. Glen. 1995. Molecular recognition of receptor sites using a genetic algorithm with a description of desolvation. J. Mol. Biol. 245:43-53.

Jones, S., and J. M. Thornton. 1996. Principles of protein-protein interactions. Proc. Natl. Acad. Sci. USA 93:13-20.

Johnson, R. E., and S. Ranganathan. 2007. Generalized approach to Ewald sums. Phys. Rev. E 75: 056706.

Jun, C-D., M. Shimaoka, C. V. Carman, J. Takagi, and T. A. Springer. 2001. Dimerization and the effectiveness of ICAM-1 in mediating LFA-1-dependent adhesion. Proc. Natl. Acad. Sci. USA 98:6830-6835.

Kabat, E. A., S. M. Beiser, and S. W. Tanenbaum. 1966. Structural similarity as seen in 3dimensional models between the N-[purin-6-oyl]-substituted amino acids and 2'-deoxyadenylic acid. Cancer Res. 26:459-460.

Kajava, A. V., and T. Vasselon. 2010. A network of hydrogen bonds on the surface of TLR2 controls ligand positioning and cell signaling.J. Biol. Chem. 285:6227-6234.

Kaplan, G., and G. Gaudernack. 1982. In vitro differentiation of human monocytes. Differences in monocyte phenotypes induced by cultivation on glass or on collagen. J. Exp. Med. 156:1101-1114.

Kaplanski G., C. Farnarier, O. Tissot, A. Pierres, A-M. Benoliel, M-C Alessi, S. Kaplanski, and P. Bongrand. 1993. Granulocyte-endothelium initial adhesion. Analysis of transient binding events mediated by E-selectin in a laminar shear flow. Biophys. J., 64:1922-1933.

Keiser, M. J., V. Setola, J. J. Irwin, C. Laggner, A. I. Abbas, S. J. Hufensein, N. H. Jensen, M. B. Kuijer, R. C. Matos, T. B. Tran, R. Whaley, R. A. Glennon, J. Hert, K. L. H. Thomas, D. D. Edwards, B. K. Shoichet, and B. L. Roth. 2009. Predicting new molecular targets for known drugs. Nature 462:175-182.

Koch, M., S. Camp, T. Collen, D. Avila, J. Salomonsen, H-J Wallny, A. van Hateren, L. Hunt, J. P. Jacob, F. Johnston, D. A. Marston, I. Shaw, P. R. Dunbar, V. Cerundolo, E. Y. Jones, and J. Kaufman. 2007. Structures of an MHC class I molecule from B21 chickens illustrate promiscuous peptide binding. Immunity 27:885-899.

Kozack, R. E., M. J. d'Mello, and S. Subramanian. 1995. Computer modeling of electrostatic steering and orientational effects in antibody-antigen association. it Biophys. J. 68:807-814. 
Kramer, A., T. Keitel, K. Winkler, W. Stocklein, W. Hohne, and J. Schneider-Mergener. 1997. Molecular basis for the binding promiscuity of an anti-p24 (HIV-1) monoclonal antibody. Cell 91:799-809.

Kramers, H. A. 1940. Brownian motion in a field of force and the diffusion model of chemical reactions. Physica VII:284-304.

Krishnan, L., G. Sahni, K. J. Kaur, and D. M. Salunke. 2008. Role of antibody paratope conformational flexibility in the manifestation of molecular mimicry. Biophys. J. 94:13671376.

Kucik, D. F., M. L. Dustin, J. M. Miller, and E. J. Brown. 1996. Adhesion-activating phorbol ester increases the mobility of leukocyte integrin LFA-1 in cultured lymphocytes. $J$. Clin. Invest. 97:2139-2144.

Kumar, R. A., J. F. Dong, J. A. Thaggard, M. A. Cruz, J. A. Lopez, and L. V. McIntire. 2003. Kinetics of GPEbalpha-vVWF-A1 tether bond under flow : effect of GPIbalpha mutations on the association and dissociation rates. Biophys. J. 85:4099-4109.

Lawrence, M. B., and T. A. Springer. 1991. Leukocytes roll on a selectin at physiologic flow rates : distinction from and prerequisite for adhesion through integrins. Cell 65:859-873.

Lee, B., and F. M. Richards. 1971. The interpretation of protein structures: Estimation of static accessibility. J. Mol. Biol. 55:379-400.

Lee, G. U., D. A. Kidwell, and R. J. Colton. 1994. Sensing discrete streptavidin-biotin interactions with atomic force microscopy. Langmuir $10: 354-357$.

Lo, C. M., H. B. Wang, M. Dembo, and Y. L. Wang. 2000. Cell movement is guided by the rigidity of the substrate. Biophys. J. 79:144-152.

Ma, Z., P. Janmey, and T. H. Finkel. 2008. The receptor deformation model of TCR triggering. FASEB. J. 22:1002-1008.

Lou, J., T. Yago, A. G. Klopocki, P. Mehta, W. Chen, V. I. Zarnitsyna, N. V. Bovin, C. Zhu, and R. P. McEver. 2006. Flow-enhanced adhesion regulated by a selectin interdomain hinge. J. Cell Biol. 174:1107-1117.

Lou J and Zhu C (2007) A structure-based sliding-rebinding mechanism for catch bonds. Biophys. J. 92:1471-1485.

Margenau, H., and N. R. Kestner. 1969. Theory of intermolecular forces. Oxford: Pergamon.

Marshall, B., M. Long, J. W. Piper, T. Yago, R. P. McEver, and C. Zhu. 2003. Direct observation of catch bonds involving cell-adhesion molecules. Nature 423:190-193.

Marshall, B. T., K. K. Sarangapani, J. Lou, R. P. McEver, and C. Zhu. .2005. Force history dependence of receptor-ligand dissociation. Biophys. J 88:1458-1466.

McFarland, B. J., and R. K. Strong. 2003. Thermodynamic analysis of degenerate recognition by the NKG2D immunoreceptor: not induced fit but rigid adaptation. Immunity 19:803-812.

McKeithan, T. W. 1995. Kinetic proofreading in T-cell receptor signal transduction. Proc. Natl. Acad. Sci. USA 92:5042-5046.

Mege, J. L., C. Capo, A. M. Benoliel, and P. Bongrand. 1987. Use of cell contour analysis to evaluate the affinity between macrophages and glutaraldehyde-treated erythrocytes. Biophys. J. 52:177-186.

Menon, S., K. Rosenberg, S. A. Graham, E. M. Ward, M. E. Taylor, K. Drickamer, and D. Leckband. 2009. Binding-site geometry and flexibility in DC-SIGN demonstrated with surface force measurements. Proc. Natl. Acad. Sci. USA 106:11524-11529.

Merkel R., P. Nassoy, A. Leung, K. Ritchie, and E. Evans. 1999. Energy landscapes of receptor-ligand bonds explored with dynamic force spectroscopy. Nature 397:50-53. 
Miller, J., R. Knorr, M. Ferrone, R. Houdei, C. P. Carron, and M. L. Dustin. 1995. Intercellular adhesion molecule- 1 dimerization and its consequences for adhesion mediated by lymphocyte function associated-1. J. Exp. Med. 182:1231-1241.

Murphy, K.,P. Travers and M. Walport. 2008. Janeway's Immunobiology. 7th edition, New York: Garland Science.

Jencks, W. P. 1981. On the attribution and additivity of binding energies. Proc. Natl. Acad. Sci. USA 78:4046-4050.

Neves, S. R., P. Tsokas, A. Sarkar, E. A. Grace, P. Rangamani, S. M. Taubenfeld, C. M. Alberini, J. C. Schaff, R. D. Blitzer, I. I. Moraru, and R. Iyengar. 2008. Cell shape and negative links in regulatory motivs together control spatial information flow in signaling networks. Cell 133:666-680.

Nishino, M., H. Tanaka, H. Ogura, Y. Inoue, T. Koh, K. Fujita and H. Sugimoto .2005. Serial changes in leukocyte deformability and whole blood rheology in patients with sepsis or trauma. J. Trauma Injury Infection Critical Care 59:1425-1431.

Nishizaka, T., H. Miyata, H. Yoshikawa, S'I. Ishiwata, and K. Kinosita. 1995. Unbinding force of a single motor molecule measured using optical tweezers. Nature 377:251-254.

Northrup SH, Erickson HP (1992) Kinetics of protein-protein association explained by brownian dynamics computer simulation. Proc. Natl. Acad. Sci. USA 89:3338-3342.

Ooi, T., M. Oobatake, G. Nemethy, and H. A. Scheraga. 1987. Accessible surface areas as a measure of the thermodynamic parameters of hydration of peptides. Proc. Natl. Acad. Sci. USA 84:3086-3090.

Page, M. I. , and W. P. Jencks. 1971. Entropic contributions to rate accelerations in enzymic and intramolecular reactions and the chelacte effect. Proc. Natl. Acad. Sci. USA 68:1678-1683.

Palecek, S. P., J. C. Loftus, M. H. Ginsberg, D. A. Lauffenburger, and A. F. Horwitz. 1997. Integrin-ligand binding properties govern cell migration speed through cell-substratum adhesiveness. Nature 385:537-539.

Palecek, S. P., A. Huttenlocher, A. F. Horwitz and D. A. Lauffenburger. 1998. Physical and biochemical regulation of integrin release during rear detachment of migratin cells. $J$. Cell Sci. 111:929-940.

Patel, K. D., M. U. Nollert, and R. P. McEver. 1995. P-selectin must extend a sufficient length from the plasma membrane to mediate rolling of neutrophils. J. Cell Biol. 131 : 1893-1902

Pereverzev, Y. V., O. Prezhdo, W. E. Thomas, and E. V. Sokurenko.2005. Distinctive features of the biological catch bond in the jump-ramp force regime predicted by the twopathway model. Phys. Rev. E 72 : 010903.

Perret E, A. M. Benoliel, P. Nassoy, A. Pierres, V. Delmas, J. P. Thiéry, P. Bongrand, and H. Feracci .2002.Fast dissociation kinetics of the recognition between individual E-cadherin fragments revealed by flow chamber analysis. EMBO J. 21:2537-2546.

Perret E., A. Leung, and E. Evans. 2004. Trans-bonded pairs of E-cadherin exhibit a remarkable hierarchy of mechanical strengths. Proc. Natl. Acad. Sci. USA 101:16472-16477.

Pierres, A., A. M. Benoliel, and P. Bongrand. 1995. Measuring the lifetime of bonds made between surface-linked molecules. J. Biol. Chem. 270:26586-26592.

Pierres A., A. M. Benoliel, P. Bongrand, and P. A. van der Merwe. 1996. Determination of the lifetime and force dependence of interactions of single bonds between surface-attached CD2 and CD48 adhesion molecules. Proc. Natl. Acad. Sci. USA 93:15114-15118.

Pierres, A., A. M. Benoliel, and P. Bongrand. 1996a. Measuring bonds between surfaceassociated molecules. J. Immunological Methods 196 : 105-120. 
Pierres, A., H. Feracci, V. Delmas, A. M. Benoliel, J. P. Thiéry, and P. Bongrand. 1998. Experimental study of the interaction range and association rate of surface-attached cadherin 11. Proc. Natl. Acad. Sci. USA 95:9256-9261.

Pierres, A., A. M. Benoliel, and P. Bongrand. 1998a. Studying receptor-mediated cell adhesion at the single molecule level. Cell Adhesion Commun. 5:375-395.

Pierres, A., A. M. Benoliel and P. Bongrand P. 2000. Cell-cell interactions. in Physical Chemistry of Biological Interfaces, e. A. Baszkin, and W. Norde., 459-522, Amsterdam: Marcel Dekker Inc.

Pierres, A., A-M. Benoliel, C. Zhu, and P. Bongrand. 2001). Diffusion of microspheres in shear flow near a wall : use to measure binding rates between attached molecules. Biophys. J. 81:25-42.

Pierres, A., A. M. Benoliel, and P. Bongrand. 2002. Cell fitting to adhesive surfaces : a prerequisite to firm attachment and subsequent events. Eur Cell Materials 3:31-45.

Pierres A, D. Touchard, A. M. Benoliel, and P. Bongrand. 2002a. Dissecting streptavidinbiotin interaction with a laminar flow chamber. Biophys. J. 82:3214-3223.

Pierres, A. J. Vitte, A-M. Benoliel, and P. Bongrand. 2006. Dissecting individual ligandreceptor bonds with a laminar flow chamber. Biophys. Rev. Letters 1:231-257.

Pierres, A., A. M. Benoliel, D. Touchard,and P. Bongrand .2008. How cells tiptoe on adhesive surfaces before sticking. Biophys. J. 94:4114-4122.

Pierres A, Benoliel AM, Bongrand P (2008) Studying molecular interactions at the single bond level with a laminar flow chamber. Cell. Mol. Bioengineering 1:247-262.

Pierres, A., V. Monnet-Corti, A-M. Benoliel, and P. Bongrand. 2009. Do membrane undulations help cells probe the world? Trends Cell Biol. 19:428-433.

Pincet, F., and J. Husson. 2005. The solution to the streptavidin-biotin paradox : the influence of history on the strength of single molecular bonds. Biophys. J. 89:4374-4381.

Puklin-Faucher, E., M. Gao, K. Schulten, and V. Vogel. 2006. How the headpiece hinge angle is opened: new insights into the dynamics of integrin activation. J. Cell. Biol. 175:349360 .

Price, N. E., N. C. Price, S. M. Kelly, and J. M. McDonnell. 2005. The key role of protein flexibility in modulating IgE interactions. J. Biol. Chem. 280:2324-2330.

Puri, K. D., S. Chen, and T. A. Springer.1998. Modifying the mechanical property and shear threshold of L-selectin adhesion independently of equilibrium properties. Nature 392:930-933.

Rapaport, D. C. 2004. The art of molecular dynamics simulation. Cambridge: Cambridge University Press. 2nd edition. 549pp

Remmerbach, T. W., F. Wottawah, J. Dietrich, B. Lincoln, C. Wittekind and J. Guck J. 2009. Oral cancer diagnosis by mechanical phenotyping. Cancer Res. 69:1728-1732.

Rich, R. L., and D. G. Myszka. 2006. Survey of the year 2005 commercial optical biosensor literature. J. Mol. Recognit. 19:478-534.

Rief, M., M. Gautel, F. Oesterhelt, J. M. Fernandez, and H. E. Gaub. 1997. Reversible unfolding of individual titin immunoglobulin domains by AFM. Science 276:1109-1112.

Robert, P., K. Sengupta, P. H. Puech, P. Bongrand, and L. Limozin. 2008. Tuning the formation and rupture of single ligand-receptor bonds by hyaluronan-induced repulsion Biophys. J. 95:3999-4012

Robert, P., L. Limozin, A. Pierres, and P. Bongrand. 2009. Biomolecule association rates do not provide a complete description of bond formation. Biophys. J. 96:4642-4650.

Rudolph, M. G., R. L. Stanfield, and I. A. Wilson. 2006. How TCRs bind MHCs, peptides and coreceptors. Annu. Rev. Immunol. 24: 419-466. 
Sabri, S., A. Pierres, A. M. Benoliel, and P. Bongrand. 1995. Influence of surface charges on cell adhesion : difference between static and dynamic conditions. Biochem. Cell Biol. 73 : 411-420.

Sabri, S., M. Soler, C. Foa, A. Pierres, A. M. Benoliel, and P. Bongrand. 2000. Glycocalyx modulation is a physiological means of regulating cell adhesion. J. Cell Sci. 113:1589-1600.

Schoen, I., H. Krammer, and D. Braun. 2009. Hybridization kinetics is different inside cells. Proc. Natl. Acad. Sci USA 106:21649-21654.

Schuck, P. 1997. Use of surface plasmon resonance to probe the equilibrium and dynamic aspects of interactions between biological macromolecules. Ann. Rev. Biophys. Biomol. Structure. 26 : 541-566.

Schueler-Furman, O., C. Wang, P. Bradley, K. Misura, and D. Baker. 2005. Progress in modeling of protein structures and interactions. Science 310:638-642.

Schwieters, C. D., J. J. Kuszewski, N. Tjandra, and G. M. Clore. 2003. The Xplor-NIH NMR molecular structure determination package.J. Magn. Res. 160:65-73.

Seifert, U. 2000. Rupture of multiple parallel molecular bonds under dynamic loading. Physical Review Letters 84:2750-2753.

Seifert, U. 2001. Dynamic strength of adhesion molecules: role of rebinding and selfconsistent rates. Europhys. Lett 58:792-798.

Shoichet, B. K., R. M. Stroud, D. V. Santi, I. D. Kuntz, K. M. Perry. 1993. Structurebased discovery of inhibitors of thymidylate synthase. Science 259:1445-1450.

Smith-Garvin, J. E., G. A. Koretzky, and M. S. Jordan. 2009. T Cell Activation Ann. Rev. Immunol. 27:591-619.

Stone, S. R., S. Dennis, and J. Hofsteenge. 1989. Quantitative evaluation of the contribution of ionic interactions to the formation of the thrombin-hirudin complex. Biochemistry. 28:6857-6863.

Sulchek, T. A., R. W. Friddle, K. Langry, E. Y. Lau, H. Albrecht, T. V. Ratto, S. J. DeNardo, M. E. Colvin, and A. Noy. 2005. Dynamic force spectroscopy of parallel individual Mucin1-antibody bonds. Proc. Natl. Acad. Sci. USA 102:16638-16643.

Tang, C-C., Y-P. Chu, and H-Y Chen. 2007. Lifetime of ligand-receptor clusters under external force. Phys. Rev. E 76:061905.

Tha, S. P., J. Shuster, and H. L. Goldsmith. 1986. Interaction forces between red cells agglutinated by antibody. IV Time and force dependence of break-up.Biophys. J. 50:11171126 .

Thomas, W. E., E. Trintchina, M. Forero, V. Vogel, and E. Sokurenko. 2002. Bacterial adhesion to target cells enhanced by shear forces. Cell 109:913-923.

Thoumine, O., P. Kocian, A. Kottelat, and J. J. Meister. 2000. Short-term binding of fibroblasts to fibronectin : optical tweezers experiments and probabilistic analysis. Eur. Biophys. J. 29:398-408.

van Oss, C. J. F. 1991. Interaction forces between biological and other polar entities in water : how many different primary forces are there ? J. Dispersion Sci. Technol. 12 : 201-219.

Vavouri, T., J. I. Semple, R. Garcia-Verdugo, and B. Lehner. 2009. Intrinsic protein disorder and interaction promiscuity are widely associated with dosage sensitivity. Cell 138:198208.

Von Andrian, U. H., J. D. Chambers, L. M. McEvoy, R. F. Bargatze, K. E. Arfors, and E. C. Butcher. (1991) Two-step model of leukocyte-endothelial cell interaction in inflammation : distinct roles for LECAM-1 and the leukocyte beta 2 integrins in vivo. Proc. Natl. Acad. Sci. USA 88:7538-7542. 
Von Andrian, U. H., S. R. Hasslen, R. D. Nelson, S. L. Erlandsen, and E. C. Butcher. 1995. A central role for microvillous receptor presentation in leukocyte adhesion under flow. Cell 82 : 989-999.

Vorup-Jensen, T., C. V. Carman, M. Shimaoka, P. Schuck, J. Svitel, and T. A. Springer. 2005. Exposure of acidic residues as a danger signal for recognition of fibrinogen and other macromolecules by integrin alphaXbeta2. Proc. Natl. Acad. Sci. USA 102:1614-1619.

Wachsstock, D. H., W. H. Schwarz, and T. D. Pollard. 1994. Cross-linker dynamics determine the mechanical properties of actin gels. Biophys. J. 66:801-809.

Walton, E. B., S. Lee, and K. J. van Vliet. 2008. Extending Bell's model: how force transducer stiffness alters measured unbinding forces and kinetics of molecular complexes. Biophys. J. 94:2621-2630.

Wang, J., K. Zhang, H. Lu, and E. Wang. 2006. Quantifying the kinetic paths of flexible biomolecular recognition. Biophys. J. 91:866-872.

Williams A. F. (1991) Out of Equilibrium. Nature 352 : 473-474.

Williams, T. E., S. Nagarajan, P. Selvaraj, and C. Zhu. 2001. Quantifying the impact of membrane microtopology on effective two-dimensional affinity. J. Biol. Chem. 276:1328313288.

Wu, L. C., D. S. Tuot, D. S. Lyons, K. C. Garcia, and M. M. Davis. 2002. Two-step binding mechanism for T-cell receptor recognition of peptide-MHC. Nature 418:552-556.

Yang, J., C. P. Swaminathan, Y. Huang, R. Guan, S. Cho, M. C. Kieke, D. M. Cranz, R. A. Mariuzza, and E. J. Sundberg. 2003. Dissecting cooperative and additive binding energetics in the affinity maturation pathway of a protein-protein interface.J. Biol. Chem. 278:50412-50421.

Yap, A. S., W. M. Brieher, M. Pruschy, and B. M. Gumbiner. 1997. Lateral clustering of the adhesive ectodomain: a fundamental determinant of cadherin function. Current Biol. 7:308-315.

Zhou ,J., L. Zhang, Y. Leng, H-K. Tsao, Y-J. Sheng, and S. Jiang. 2006. Unbinding of the streptavidin-biotin complex by atomic force microscopy : a hybrid simulation study. $J$. Chem. Phys. 125:104905.

Zhu, C., M. Long, S. E. Chesla, and P. Bongrand .2002. Measuring receptor/ligand interaction at the single-bond level : experimental and interpretative issues. Ann. Biomed. Engineering 30:305-314.

Zhu, J., B-H. Luo, T. Xiao, C. Zhang, N. Nishida, and T. A. Springer. 2008. Structure of a complete integrin ectodomain in a physiologic resting state and activation and deactivation by applied forces. Mol. Cell 32:849-861.

Zidovska, A., and E. Sackmann. 2006. Brownian motion of nucleated cell envelopes impedes adhesion. Phys. Rev. Letters 96:048103.

Zwanzig, R. 1988. Diffusion in a rough potential.Proc. Natl. Acad. Sci. USA 85:20292030 . 